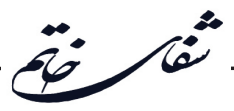

\title{
Contribution of N170 Component in Subjective Time Distortions of Facial Emotions
}

\section{Maryam Hoseini Houripasand, Mohammad Ali Nazari*, Hassan Sabouri Moghaddam, Jalil Babapour}

Department of Cognitive Neuroscience, Faculty of Psychology and Educational Sciences, University of Tabriz, Tabriz, Iran Article Info:

\section{ABSTRACT}

Introduction: Emotional effects on human time perception are generally attributed to the arousal factor, which is hypothesized to be responsible for speeding up or slowing down the internal clock. The aim of the present study was to investigate the arousal effect on time distortion with the help of event-related potentials (ERPs) that can provide direct electrophysiological $\vdots$ measures of arousal rather than self-report indices which have been the common approach in the previous studies. In this study, a temporal bisection task was employed to assess the temporal responses of participants to emotional faces that varied in arousal dimension. The N170 component was used as an objective measure of induced arousal by different emotional faces. Materials and Methods: Electroencephalography was recorded in 20 participants who performed a temporal bisection task using high arousal pleasant stimuli (happy faces) and neutral ones as stimuli. Results: Behavioral data revealed that temporal bisection points were significantly smaller for high arousal pleasant stimuli (happy faces) compared to neutral ones, while changes in N170 amplitude were independent of the emotional modulation of facial stimuli. Conclusion: In the light of the dual-stage theory of emotional processing, it seems that the N170 component is not appropriate to measure the arousal index of emotional faces in implicit tasks, and probably later components may be a better indicator for this index.

\section{Keywords:}

1. Facial Expression

2. Time Perception

3. Arousal

*Corresponding Author: Mohammad Ali Nazari

Email:nazaripsycho@yahoo.com 
نقش مولفهُ +NIV در اعوجاج زمان ذهنى ناشى از جهرههاى هيجانى

مريم حسينى حورى يسند، محمدعلى نظرى"، حسن صبورىمقدم، جليل بابايور

بخش علوم اعصاب شناختى، دانشكده روانشناسى و علوم تربيتى، دانشاه تبريز، تبريز، ايران

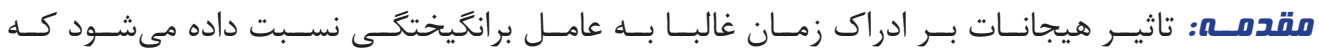

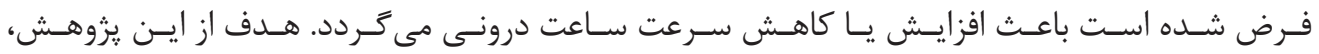

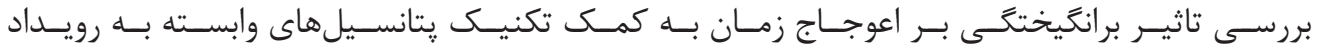

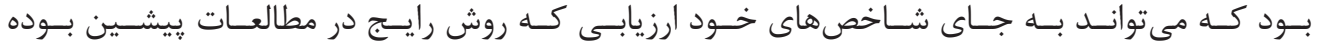

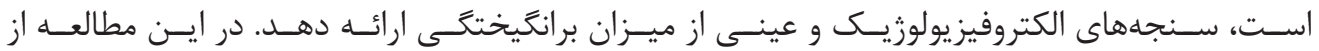

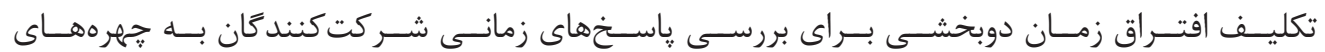

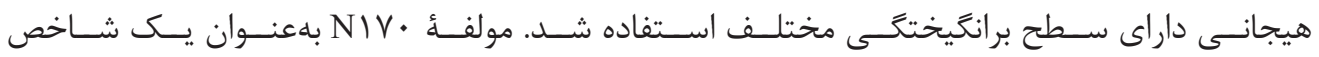

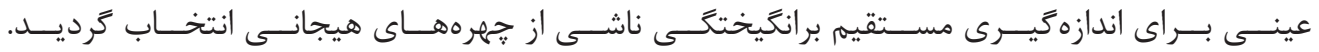

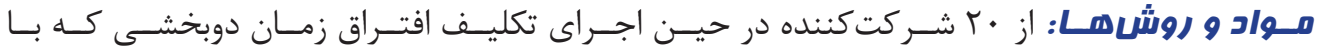

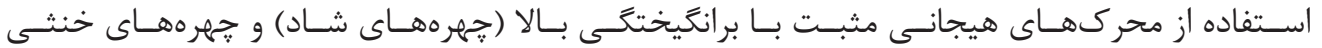

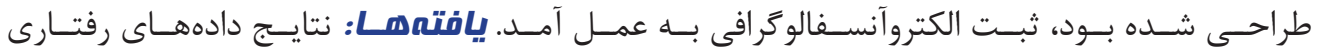

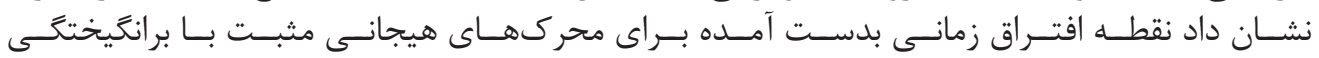

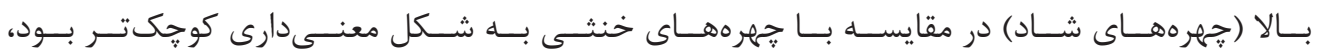

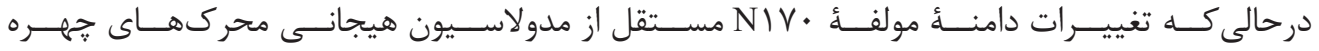
وازمهاى كليدى:

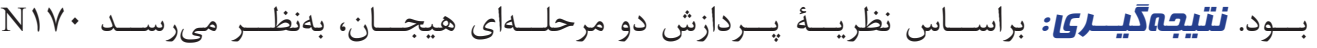

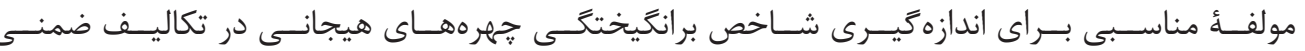
ا ب

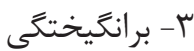

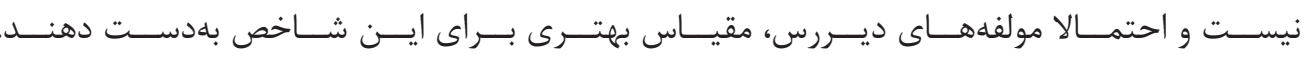




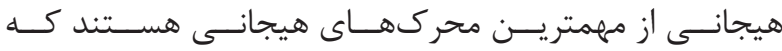

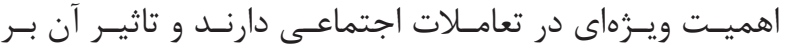

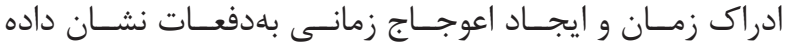

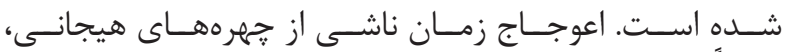

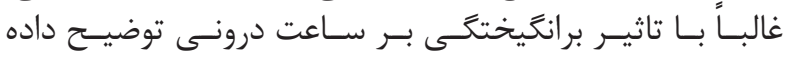

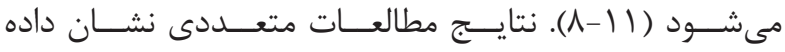

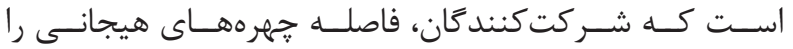

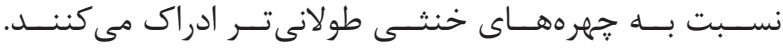

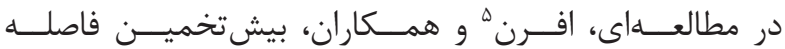

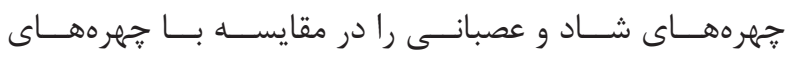

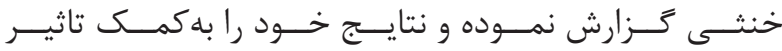

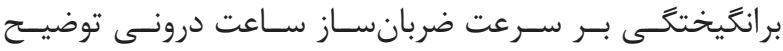

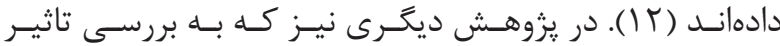

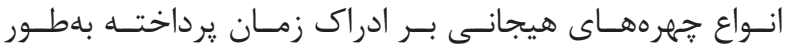

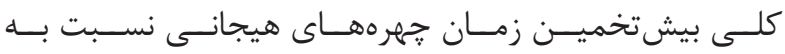

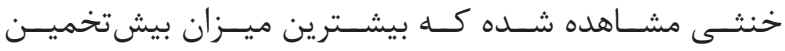

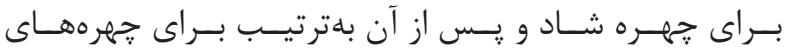

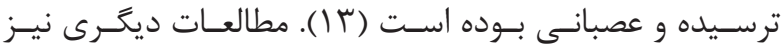

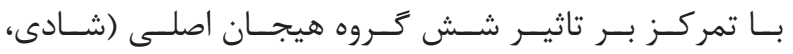

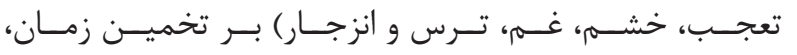

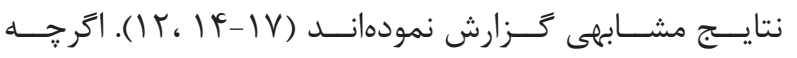

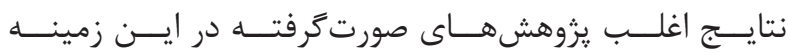

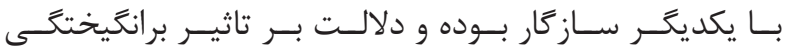

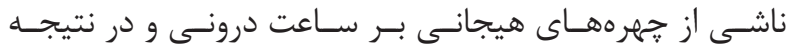

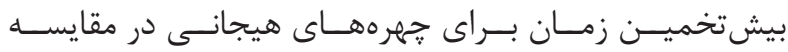

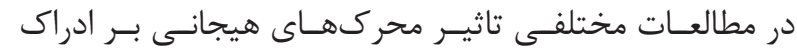

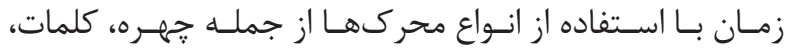

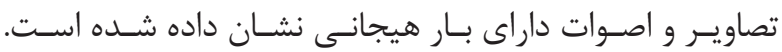

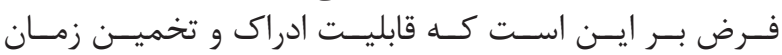

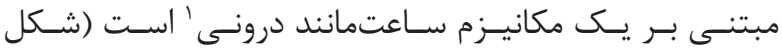

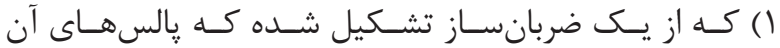

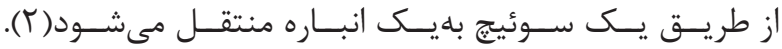

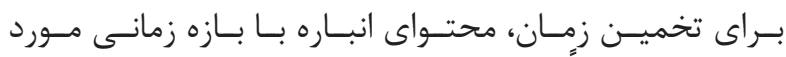

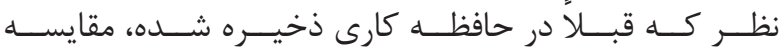

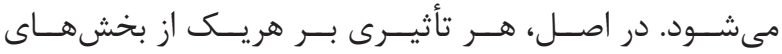

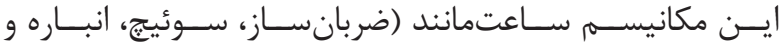

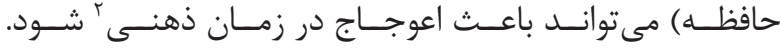

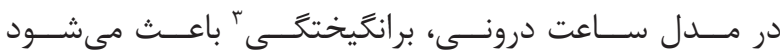

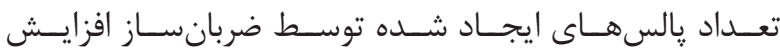

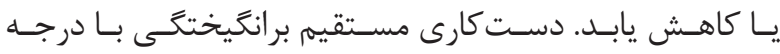

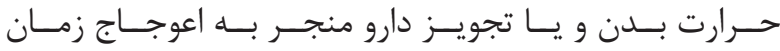

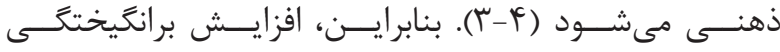

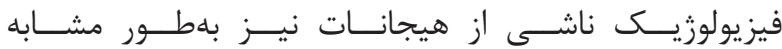

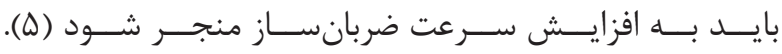

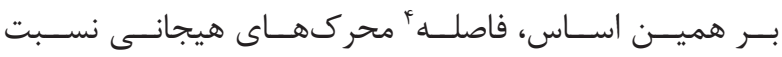

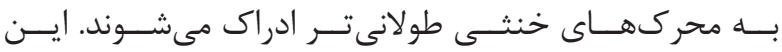

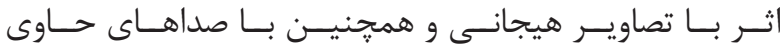

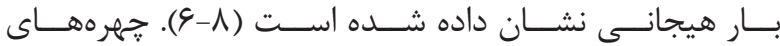

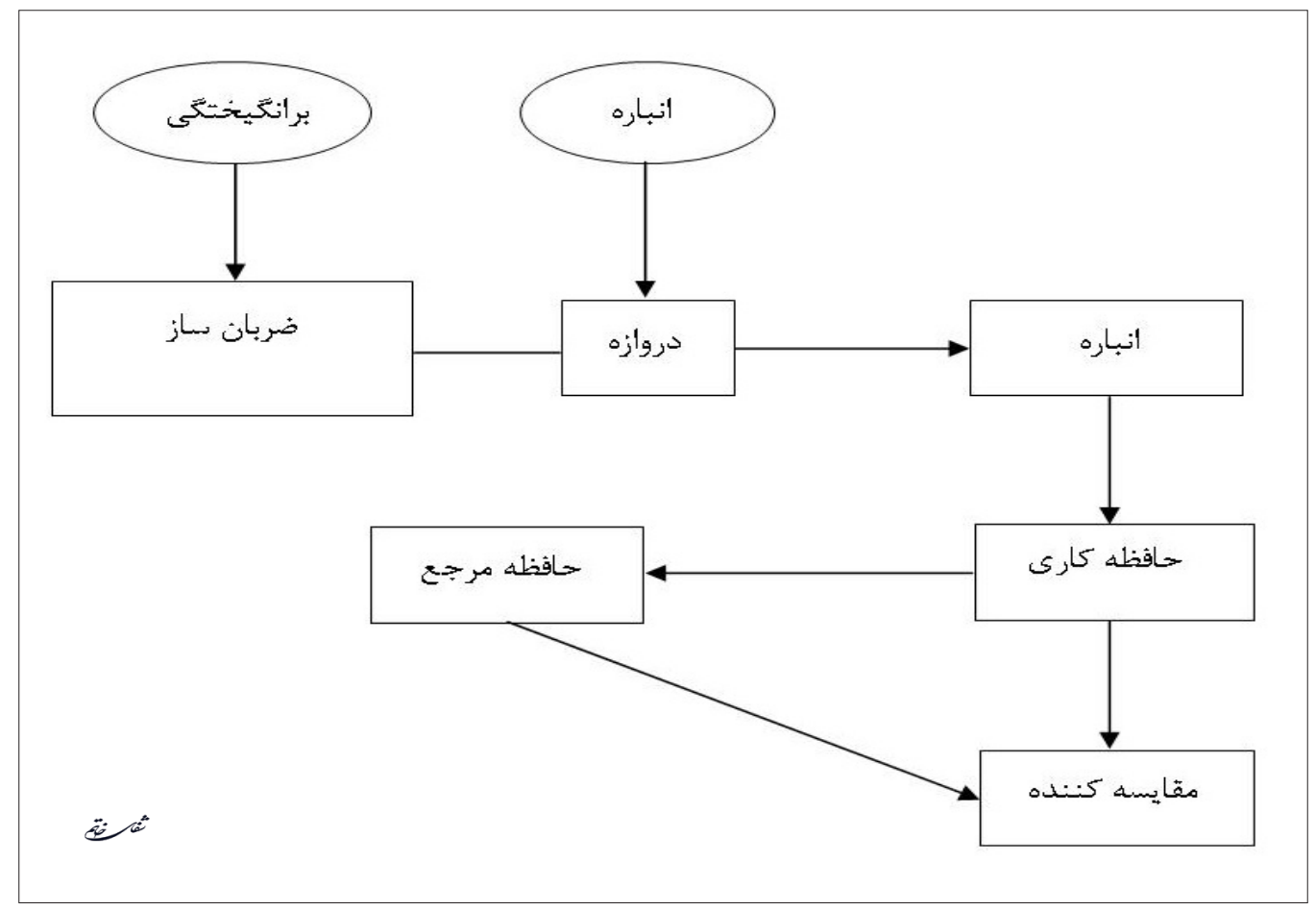

تصوير 1- ساختار مدل ساعت درونى (r)

${ }^{1}$ Internal Clock

${ }^{2}$ Subjective time Distortion

${ }^{3}$ Arousal
${ }^{4}$ Duration

${ }^{5}$ Effron 


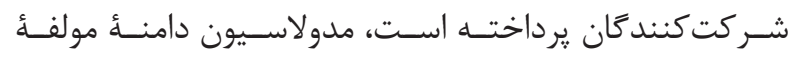

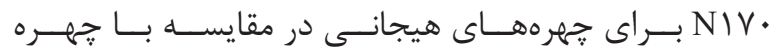

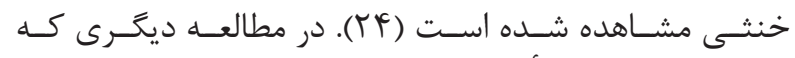

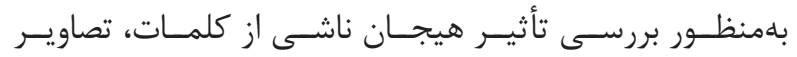

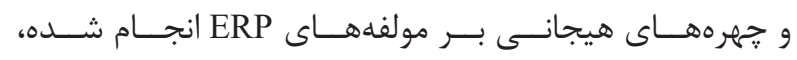

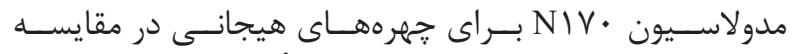

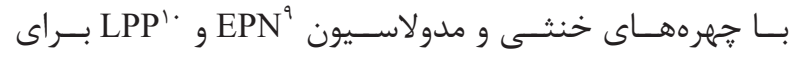

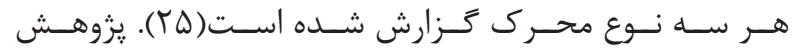

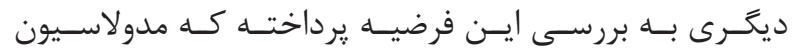

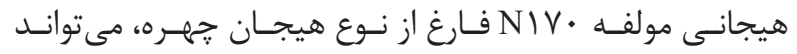

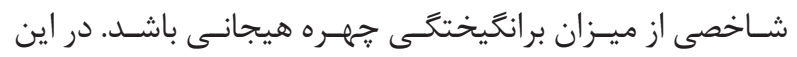

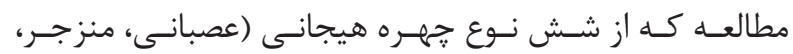

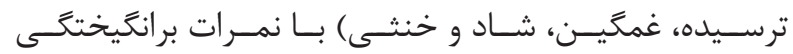

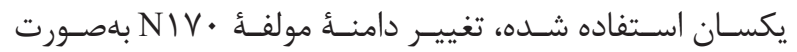

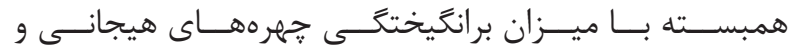

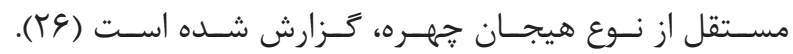

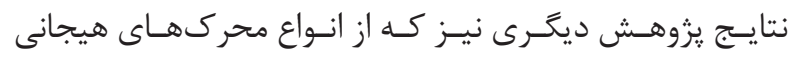

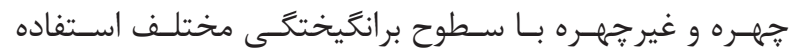

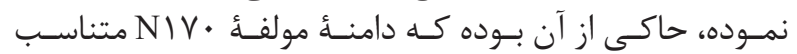

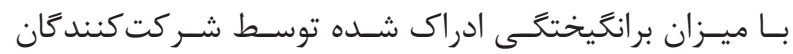

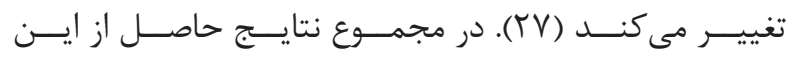

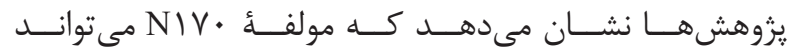

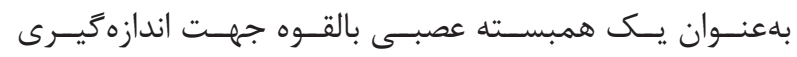

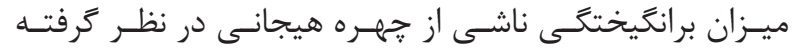

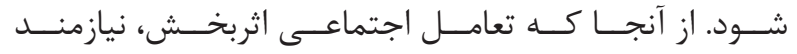

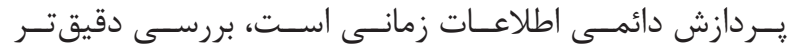

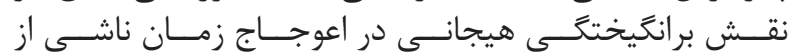

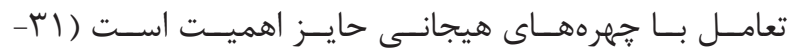

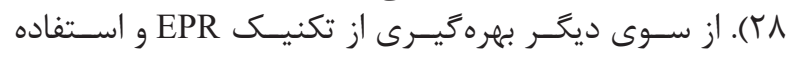

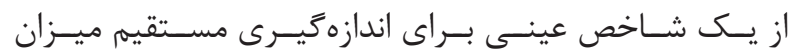

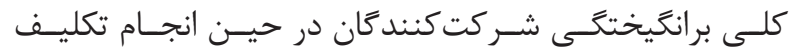

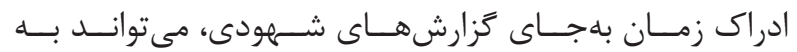

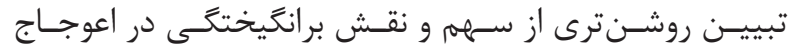

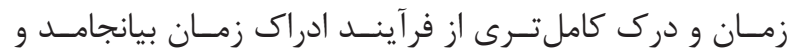

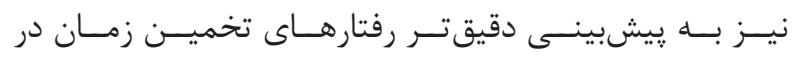

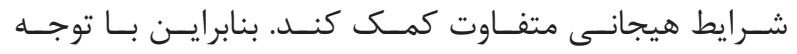

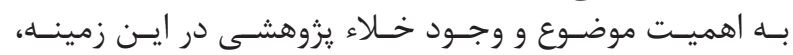

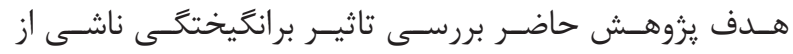

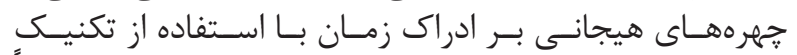

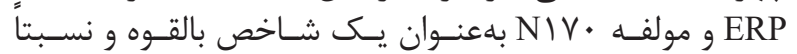

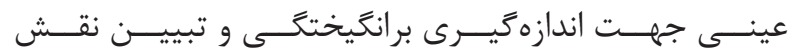

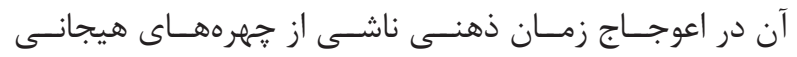

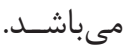

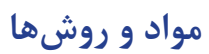

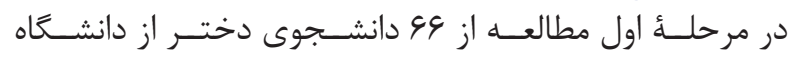

${ }^{6}$ Event Related Potential (ERP)

${ }^{7}$ Inferior-Temporal Gyrus

${ }^{8}$ Fusiform Gyrus

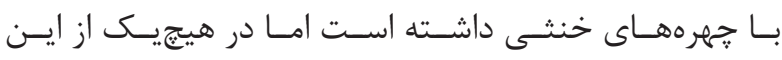

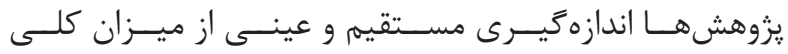

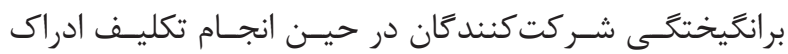

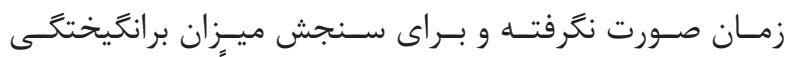

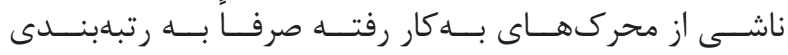

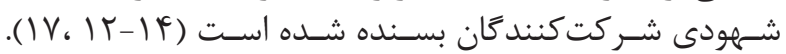

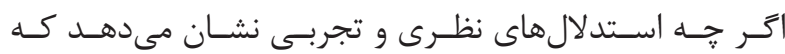

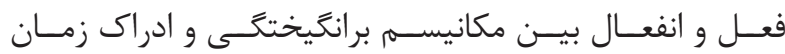

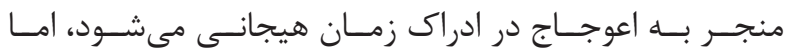

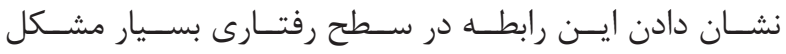

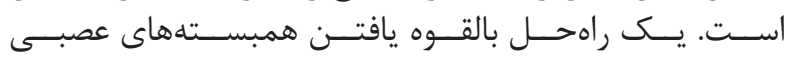

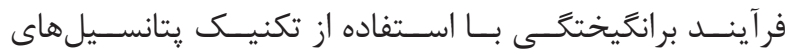

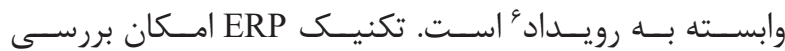

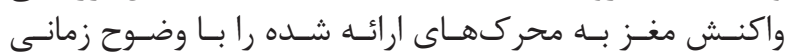

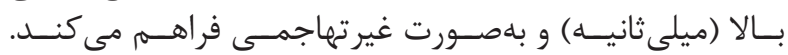

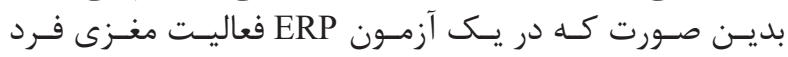

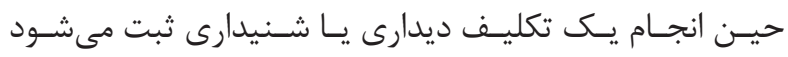

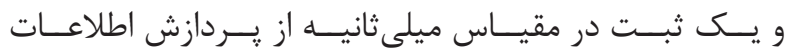

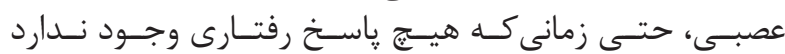

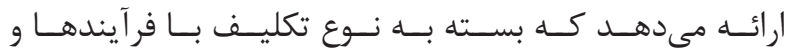

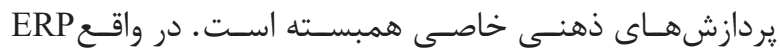

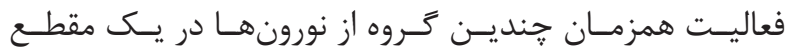

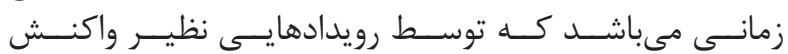

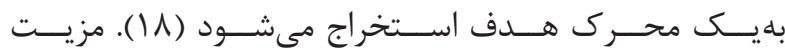

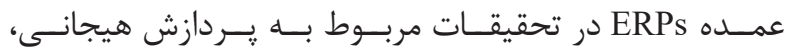

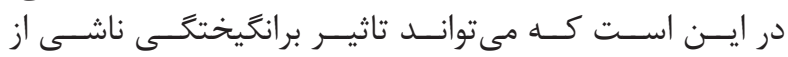

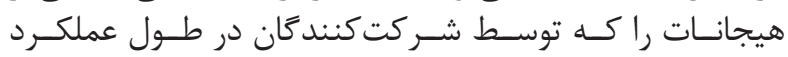

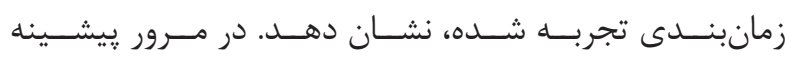

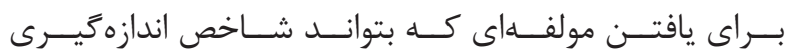

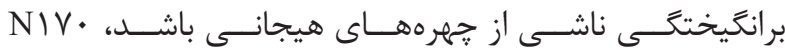

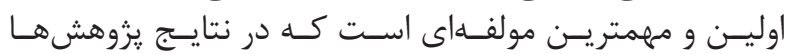

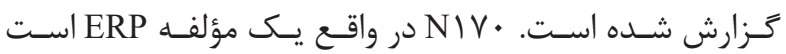

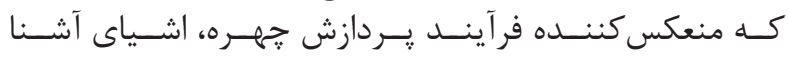

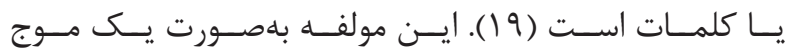

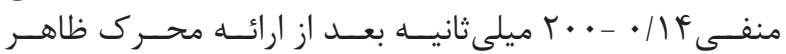

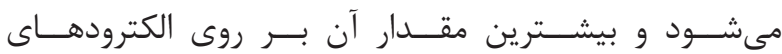

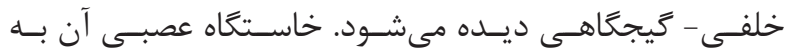

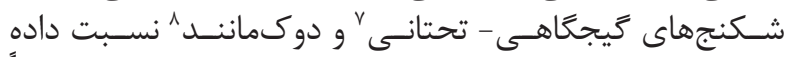

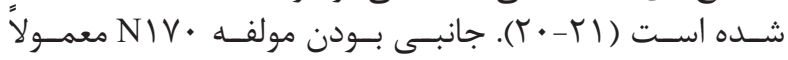

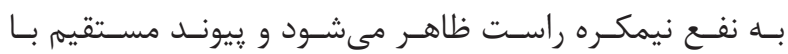

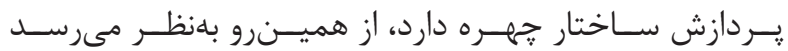

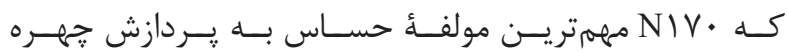

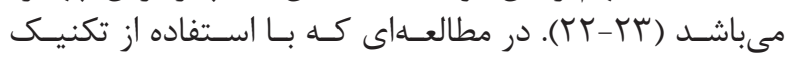

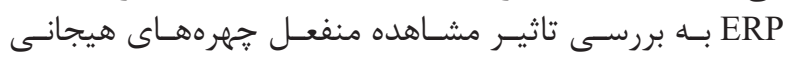

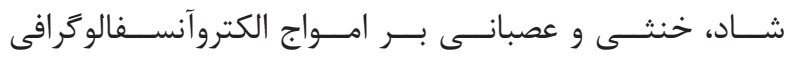

${ }^{9}$ Early Posterior Negativity (EPN)

${ }^{10}$ Late Positive Potential (LPP) 


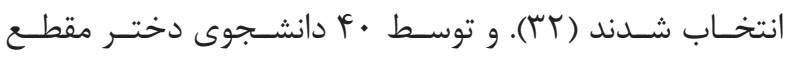

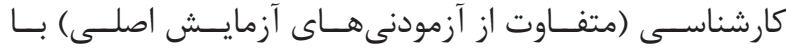

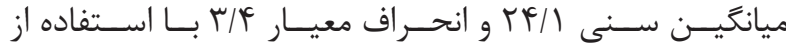

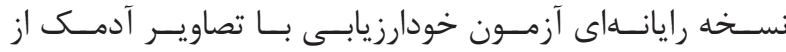

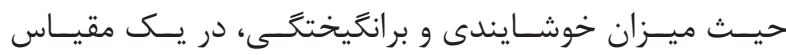

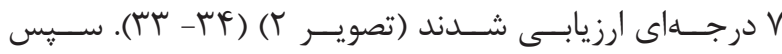

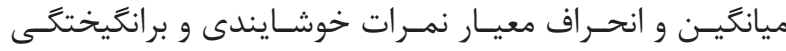

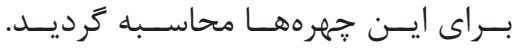

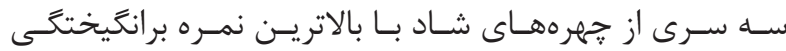

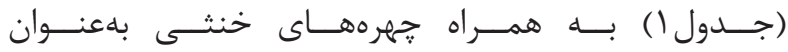

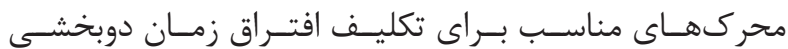

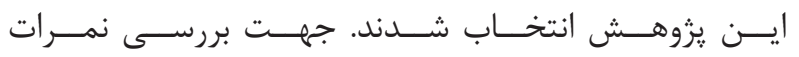

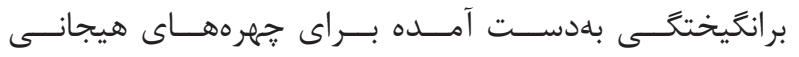

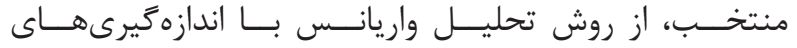

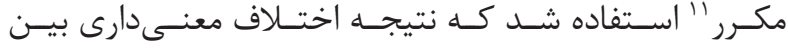

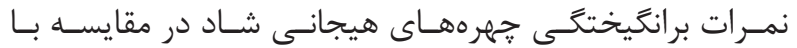

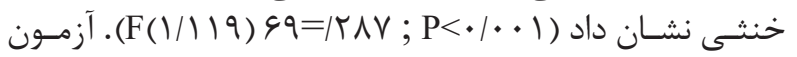

آزاد اسـلامى تهـــران واحـــد علــوم و تحقيقــات، كـهـ داوطلـب

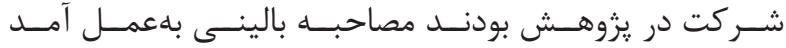

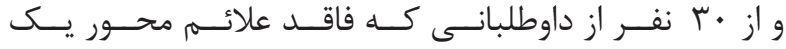

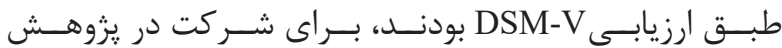

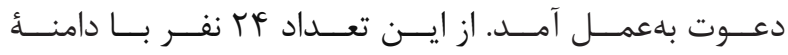

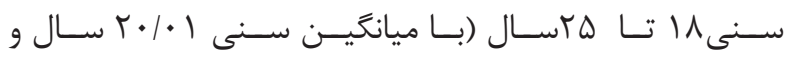

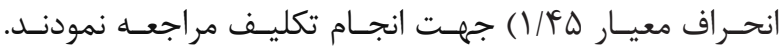

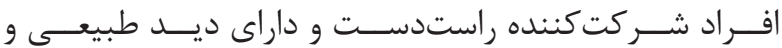

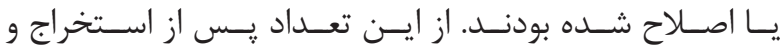

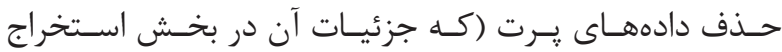

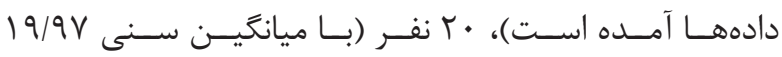

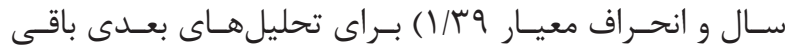

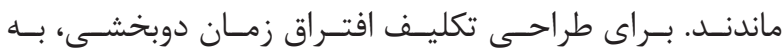

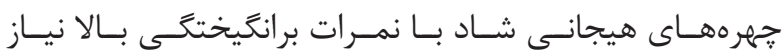

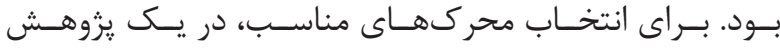

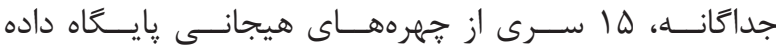

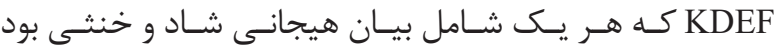

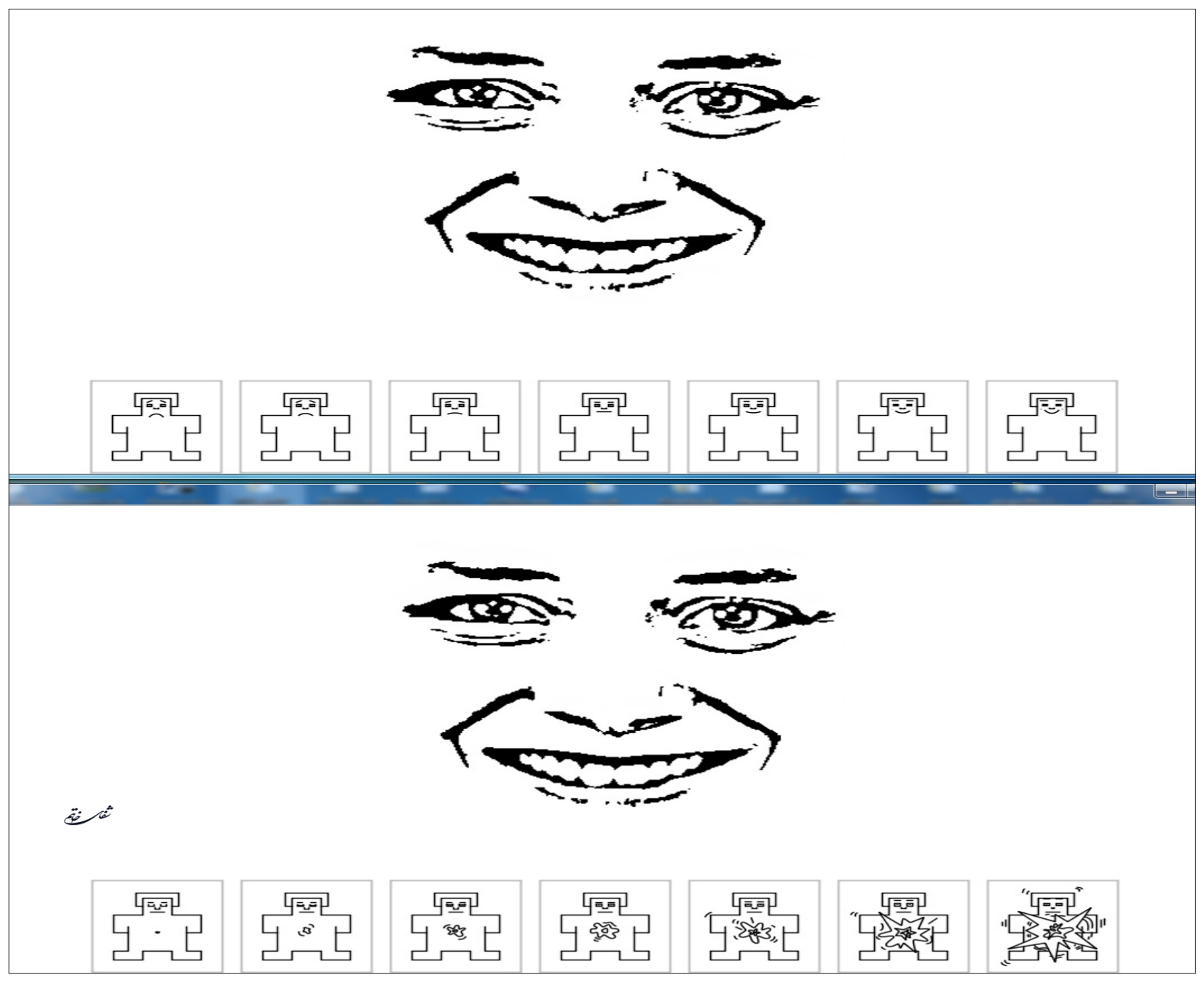

تصوير r- نمونهاى از آزمايههاى نسخه رايانهاى آزمون خودارزيابى با تصاوير آدمك SAM براى ارزيابى ميزان خوشايندى و برانكَيختكى جهره 


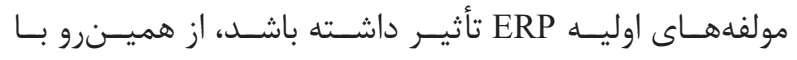

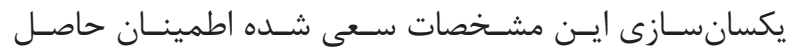

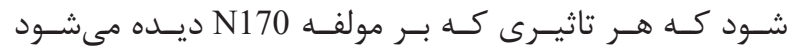

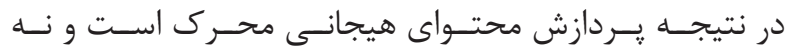

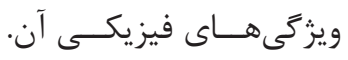

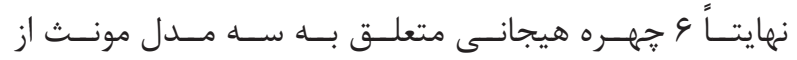

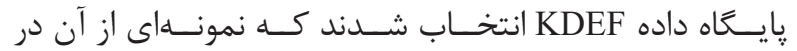

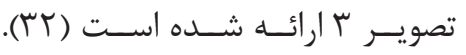

در طــول اجــراى تكليـف، شـركت كنـند

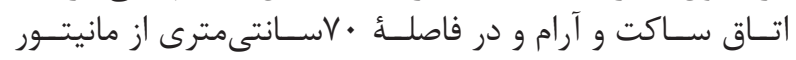

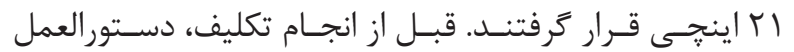

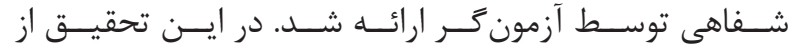

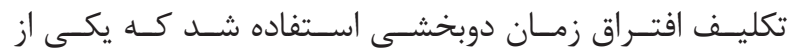

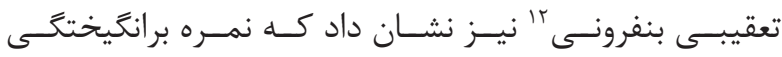

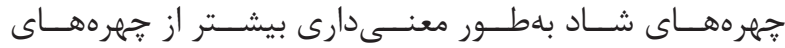

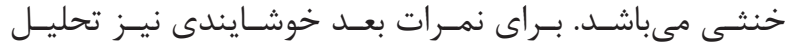

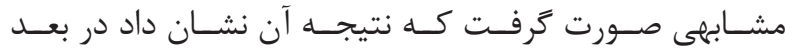

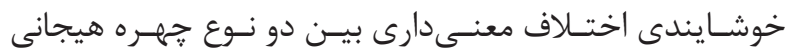

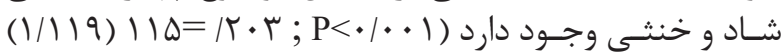
F

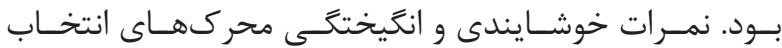

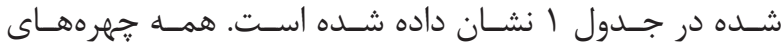

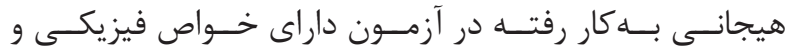

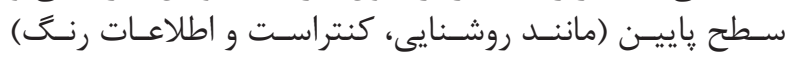

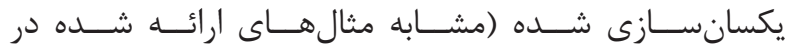

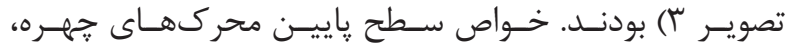

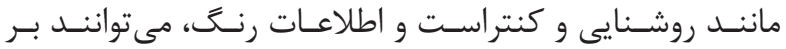

جدول ا- ميانكَين و انحراف معيار نمرات برانكَيختىى و خوشايندى محر كهاى جهره

\begin{tabular}{|c|c|c|c|c|}
\hline \multicolumn{2}{|c|}{ خوشاينلى } & \multicolumn{2}{|c|}{ برانكيختكى } & \multirow{2}{*}{ هيجان } \\
\hline انحراف معيار & ميانتين & انحراف معيار & ميانكّين & \\
\hline .1110 & $\Delta / \Delta \vee \Delta$ & $\cdot \| f \wedge$ & $f / 99 \mathrm{~V}$ & شاد \\
\hline$\cdot 1 \cdot 19$ & H/AIV & $\cdot / 1 f \Delta$ & r/MTD & خنثى \\
\hline
\end{tabular}
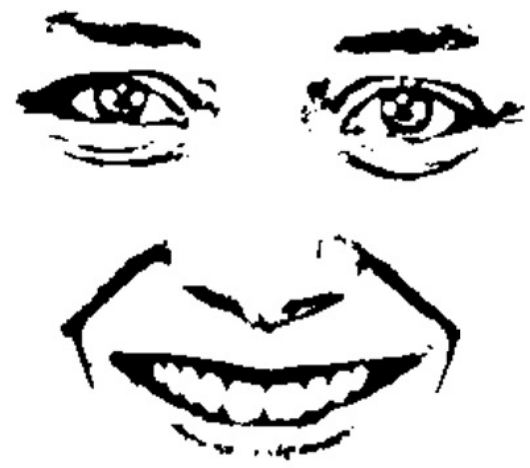

شاد
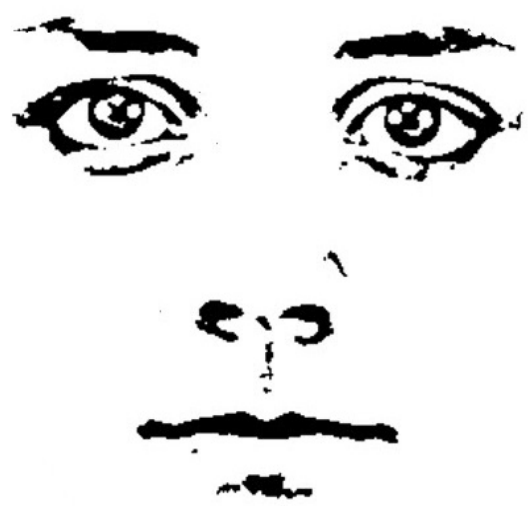

خنثى

ثمث 


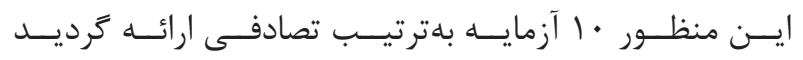

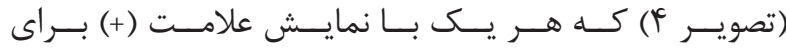

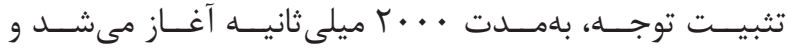

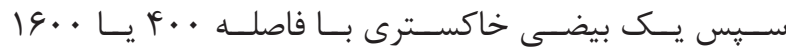

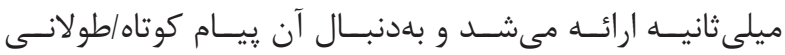

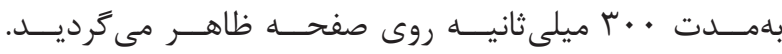

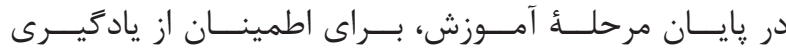

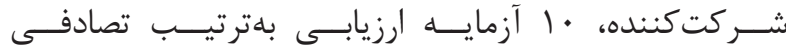

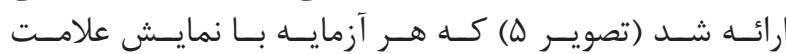

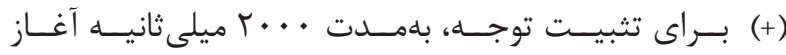

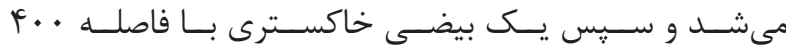

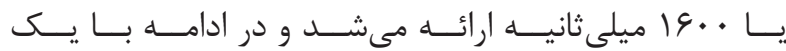

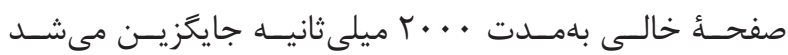

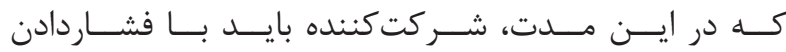

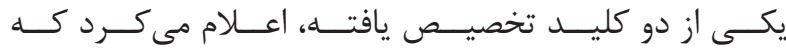

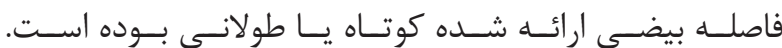

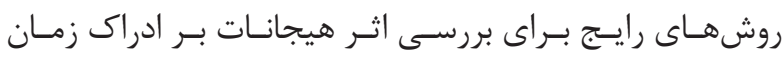

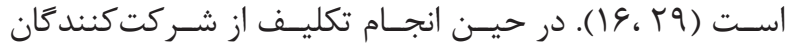

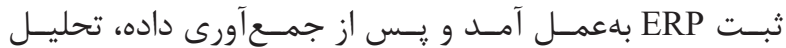

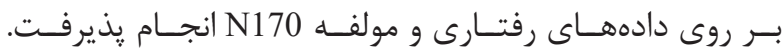

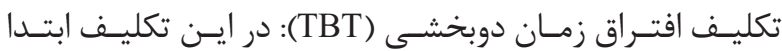

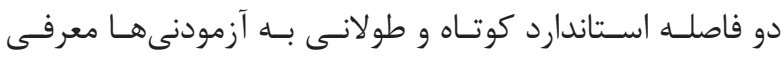

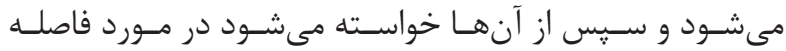

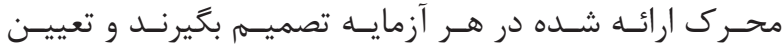

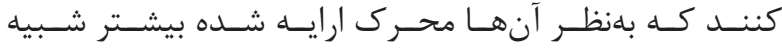

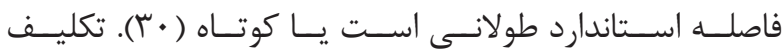

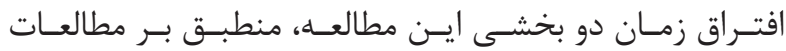

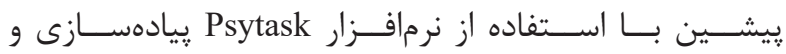

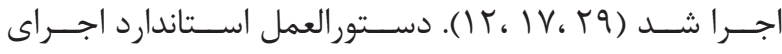

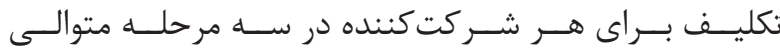

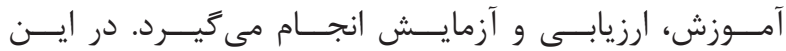

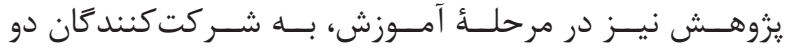

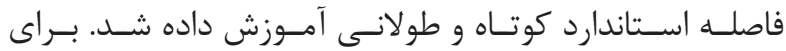

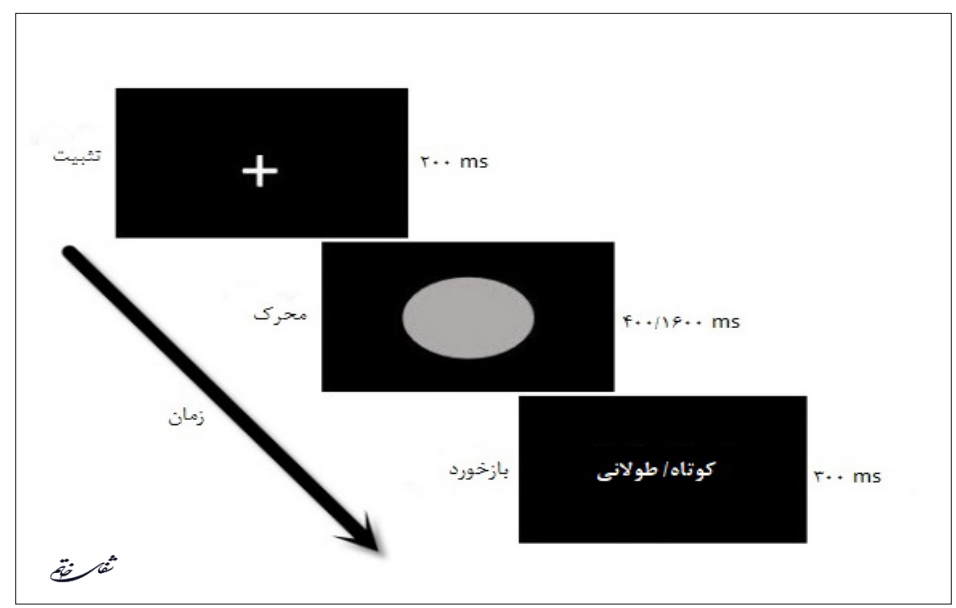

تصوير \& - توالى ارائه محركها در مرحلئ آموزش تكليف افتراق زمان دوبخشى

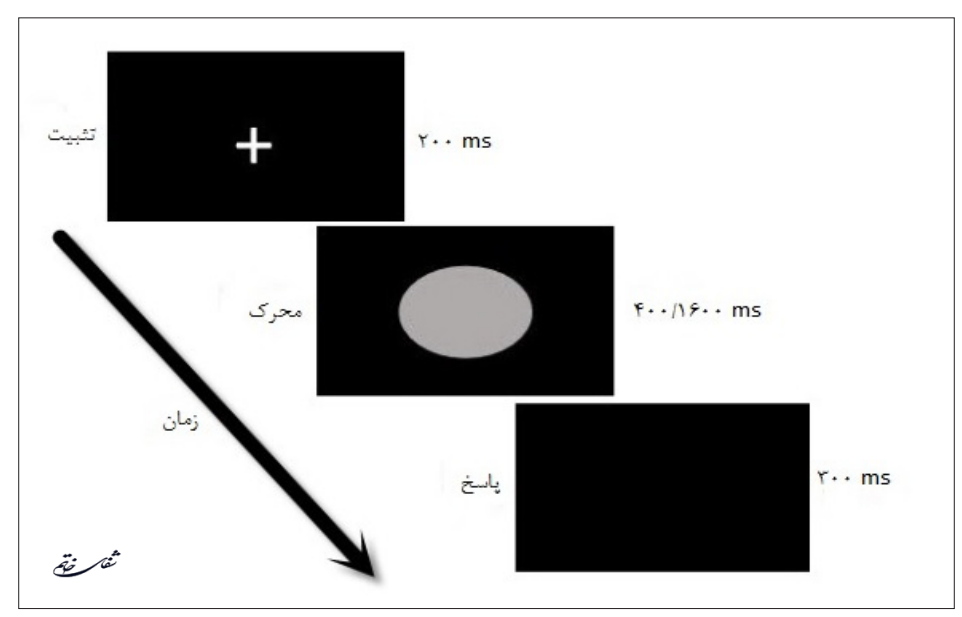

تصوير ه- توالى ارائه محركها در مرحلئ ارزيابى تكليف افتراق زمان دوبخشى 


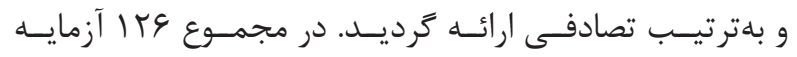

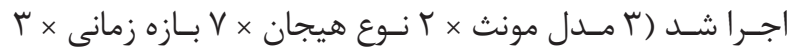

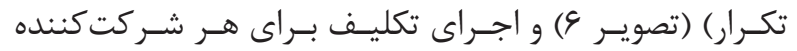

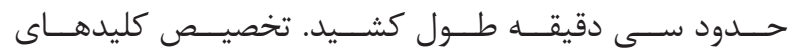

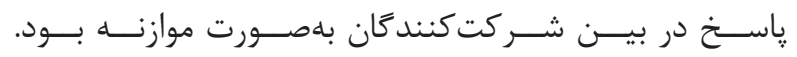

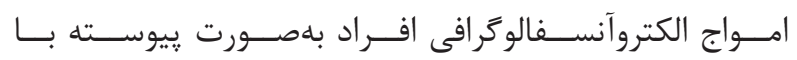

زمانى كــه شــركت كننده ايـنـ ارزيابـى را بــا موفقيـت كامــل

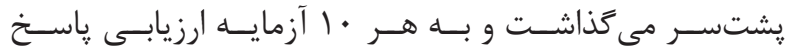

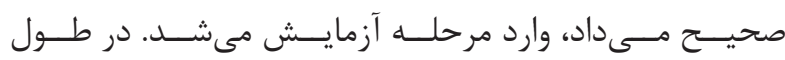

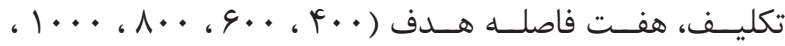

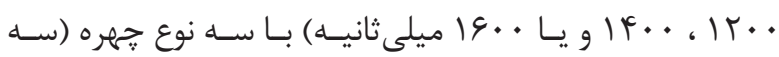

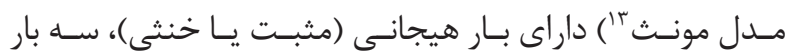

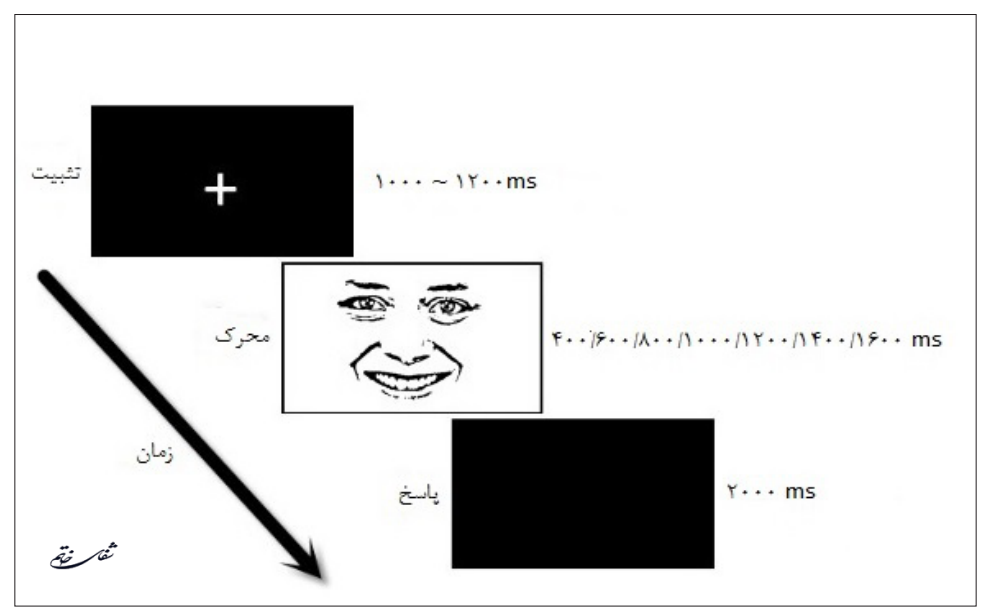

تصوير צ- توالى ارائه محر كها در مرحلة آزمون تكليف افتراق زمان دوبخشى

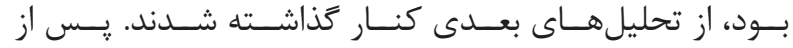

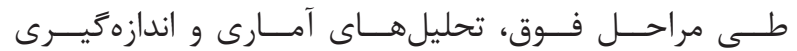

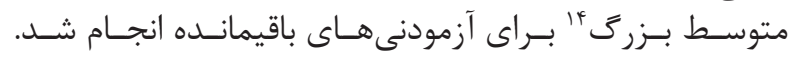

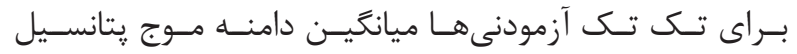

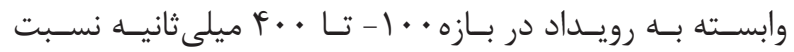

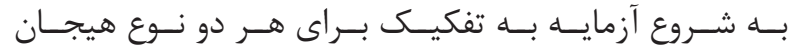

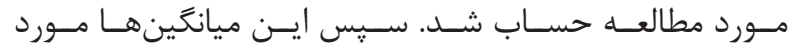

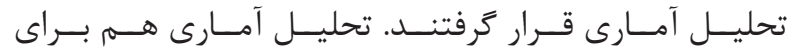

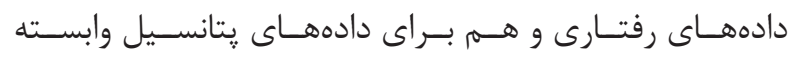

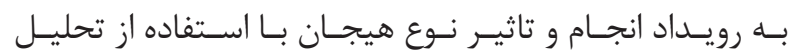

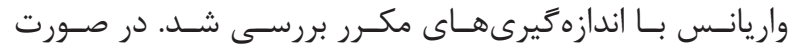

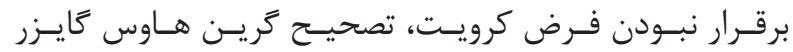

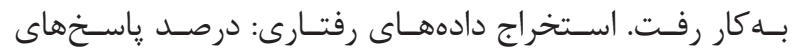

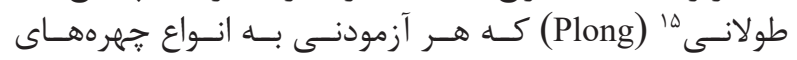

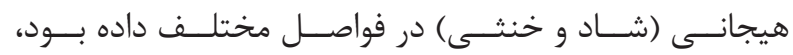

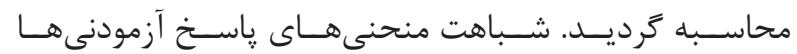

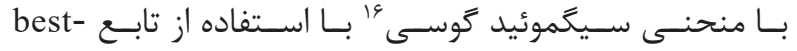

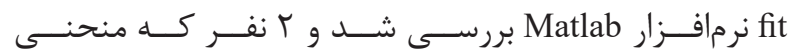

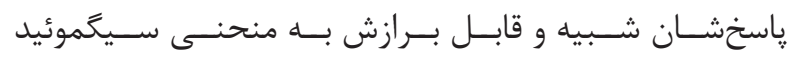

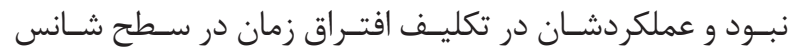

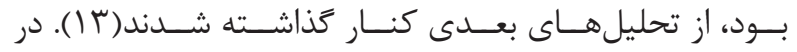

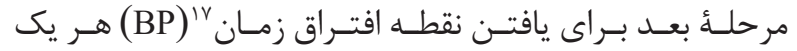

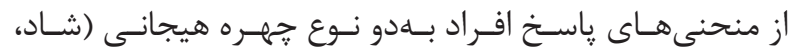

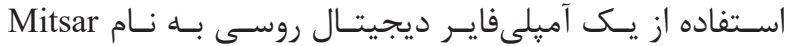

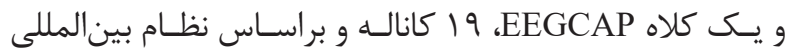

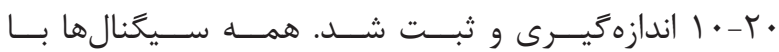

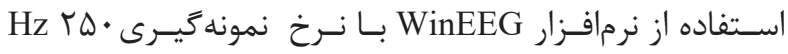

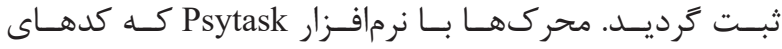

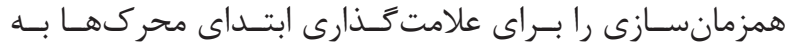

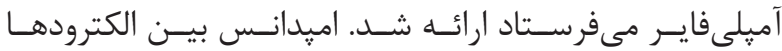

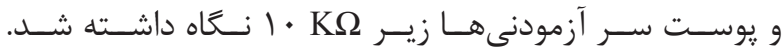

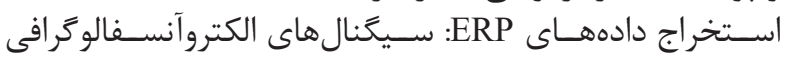

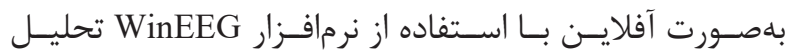

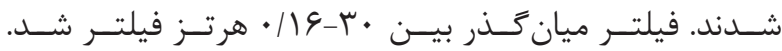

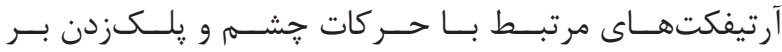

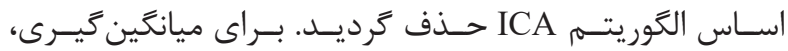

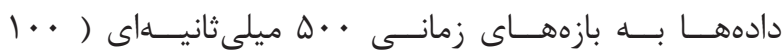

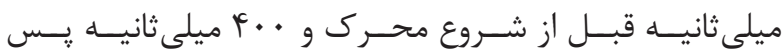

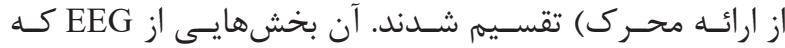

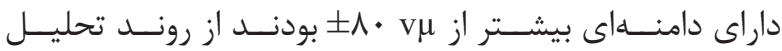

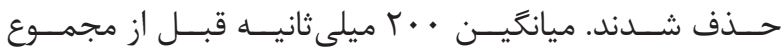

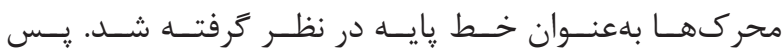

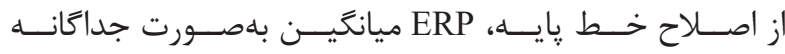

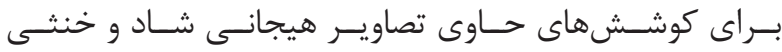

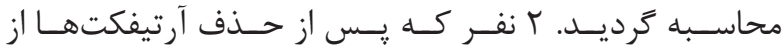

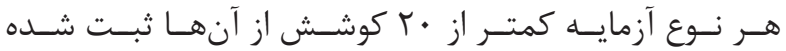




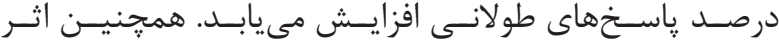

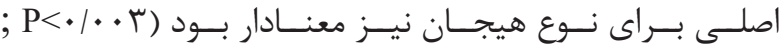

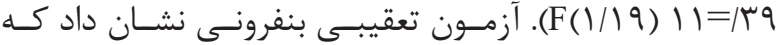

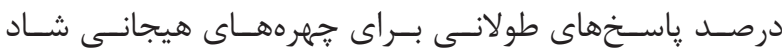

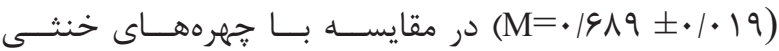

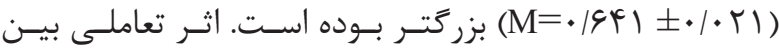

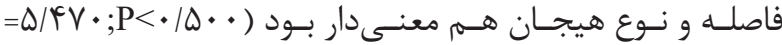

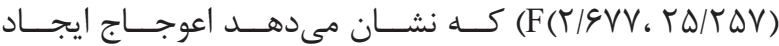

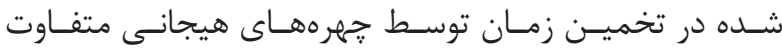

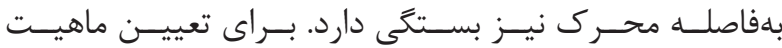

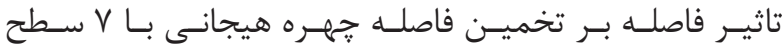

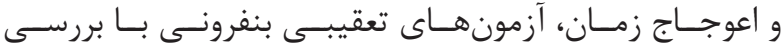

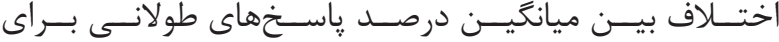

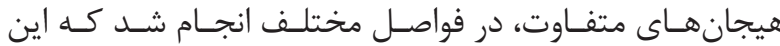

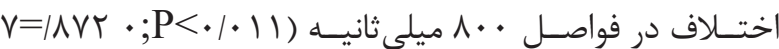

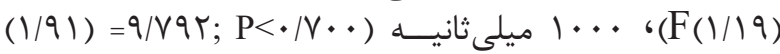
) و • F

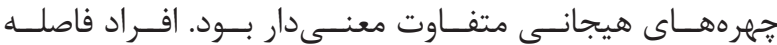

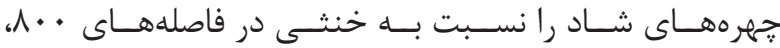

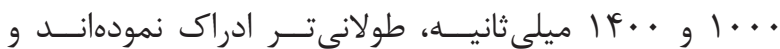

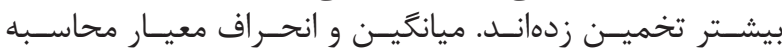

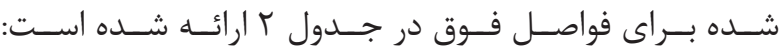

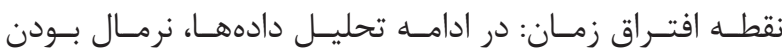

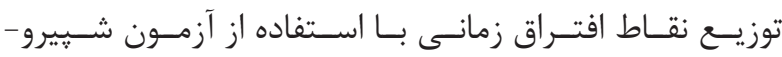

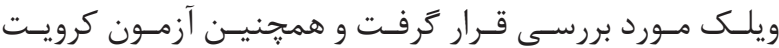

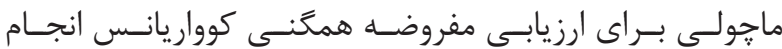

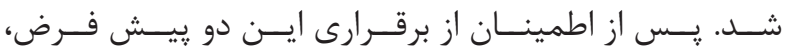

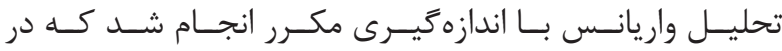

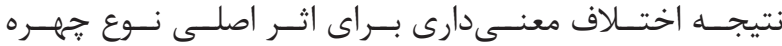

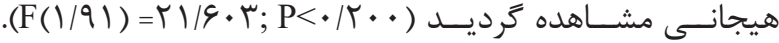

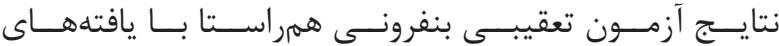

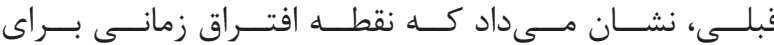

\begin{tabular}{|c|c|c|c|c|}
\hline \multicolumn{2}{|c|}{ جهره خنثى } & \multicolumn{2}{|c|}{ جهره شاد } & \multirow[b]{2}{*}{ هيجان } \\
\hline انحراف معيار & ميانتين & انحراف معيار & ميانكين & \\
\hline$\cdot / \cdot \Delta \Lambda$ & . $10+4$ & $\cdot 1 \cdot \Delta \Delta$ & $\cdot / V \cdot r$ & A.. \\
\hline $.1 \cdot 48$ & $\cdot / V \vee \wedge$ & $.1 \cdot T \Delta$ & .19 & $1 \ldots$ \\
\hline$\cdot 1 \cdot r 1$ & $.19 \Delta f$ & .1 .14 &.$/ 914$ & IF.. \\
\hline
\end{tabular}

جدول r- ميانكَين و انحراف معيار درصد پِاسخهاى طولانى براى انواع هيجان

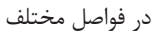

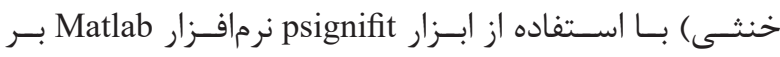

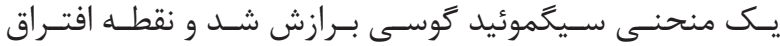

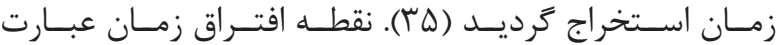

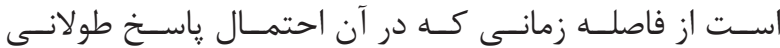

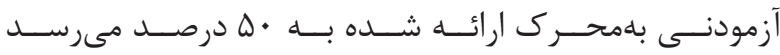

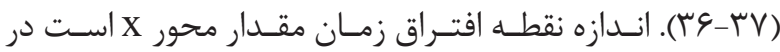

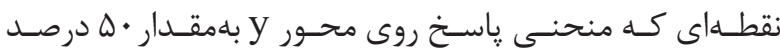

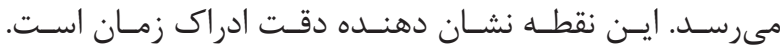

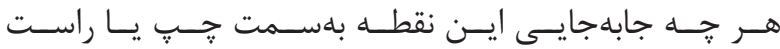

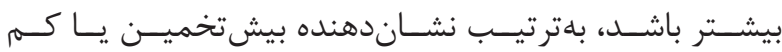

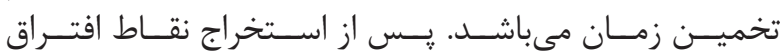

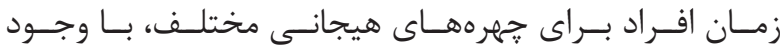

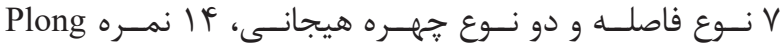

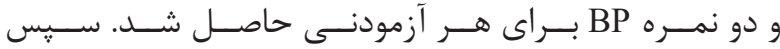

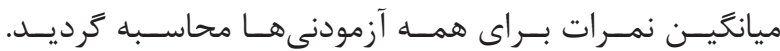

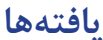

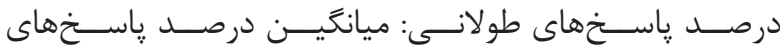

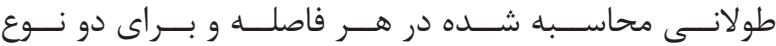

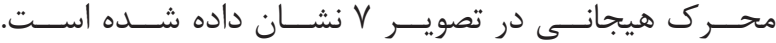

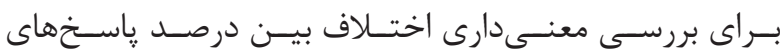

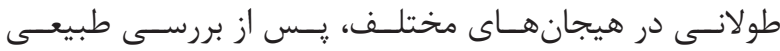

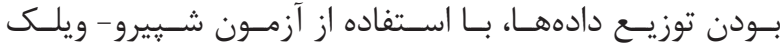

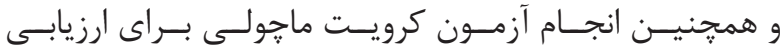

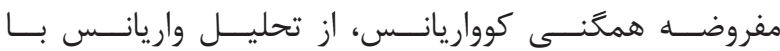

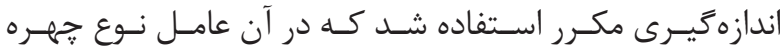

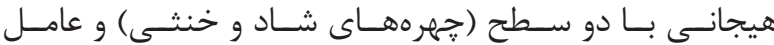

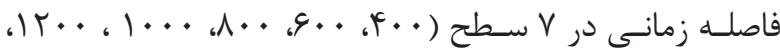

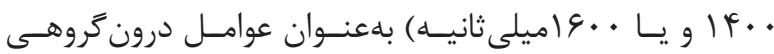

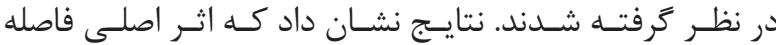
مه

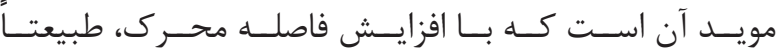

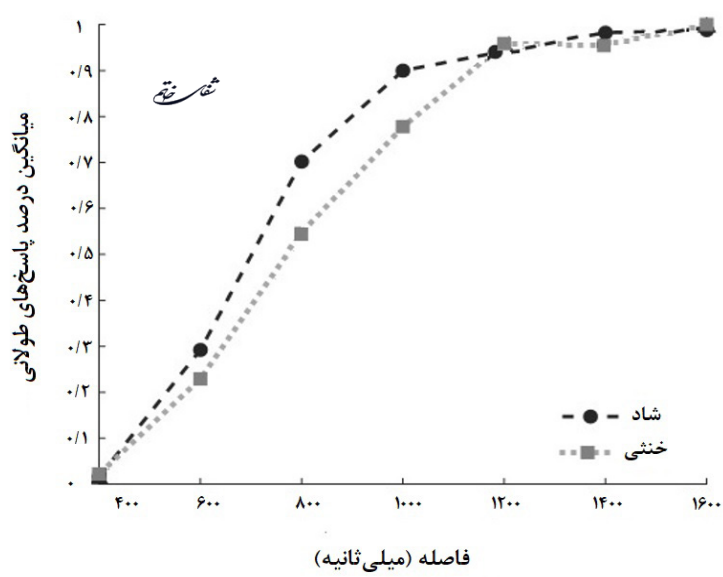

تصوير V- درصد ياسخهاى طولانى براى سه نوع جهره هيجانى (شاد و خنثى) 


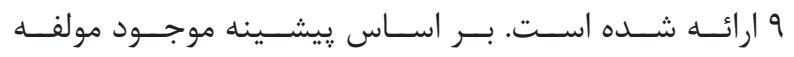

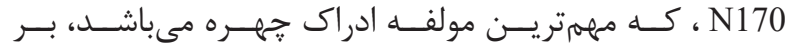

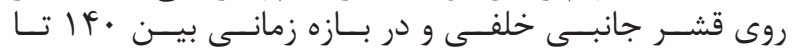

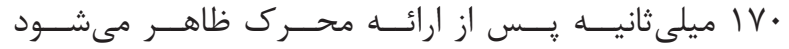

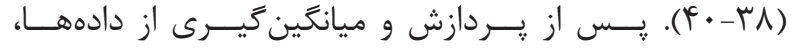

جهرههــاى شــاد (M= VYF/9 ms

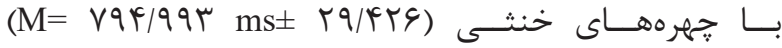

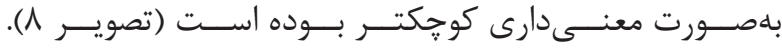
توزيــع يتانســيل روى ســر بــراى مولفــه • در تصويــــ

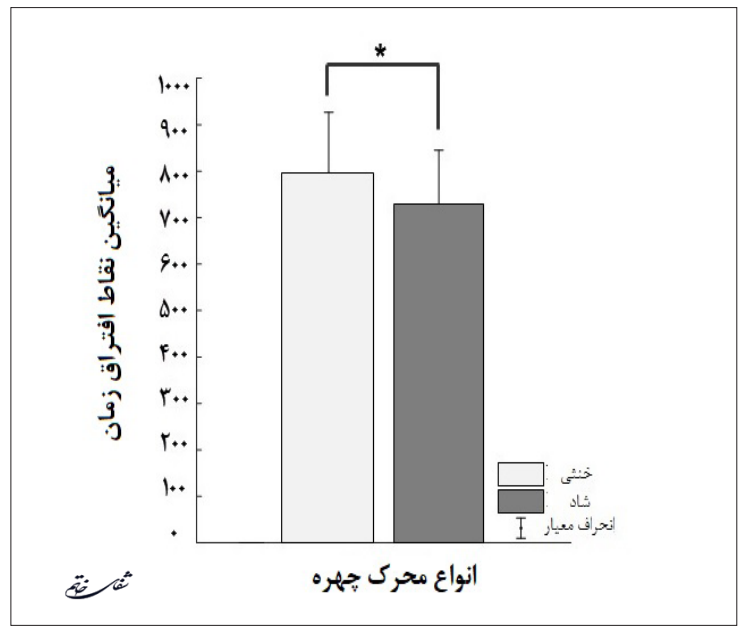

تصوير ^- ميانكين و انحراف معيار نقطه افتراق زمان براى انواع جهرههاى هيجانى (شاد و خنثى) (1)

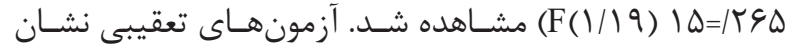

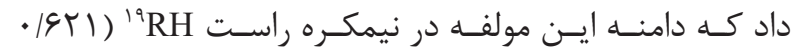

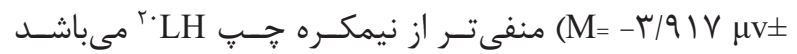

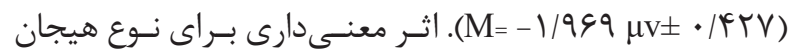

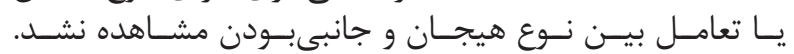

\section{بحث و نتيجه}

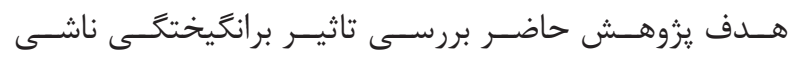

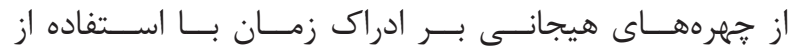

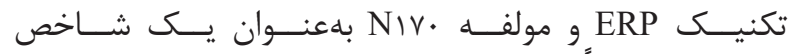

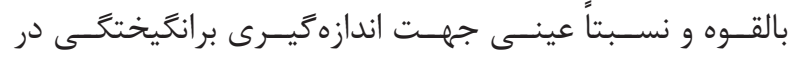

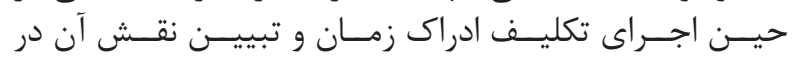

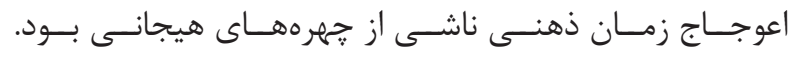

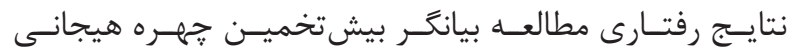

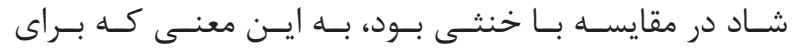

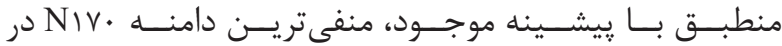

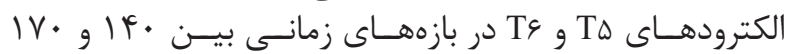

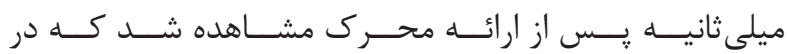

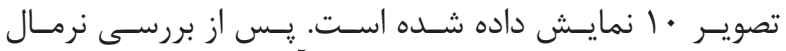

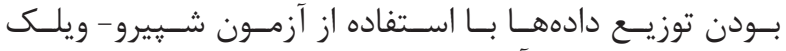

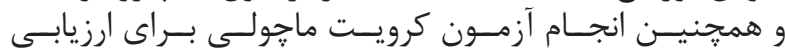

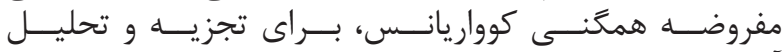

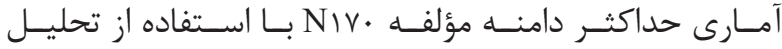

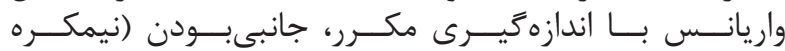

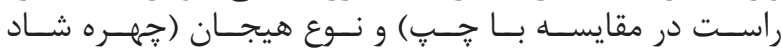

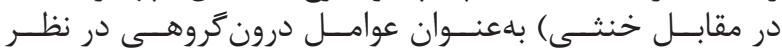

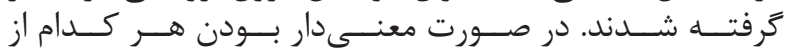

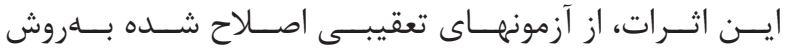

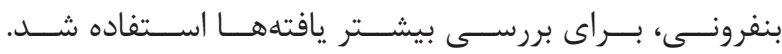

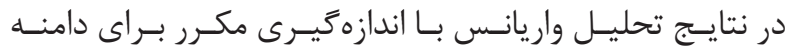
NIV.

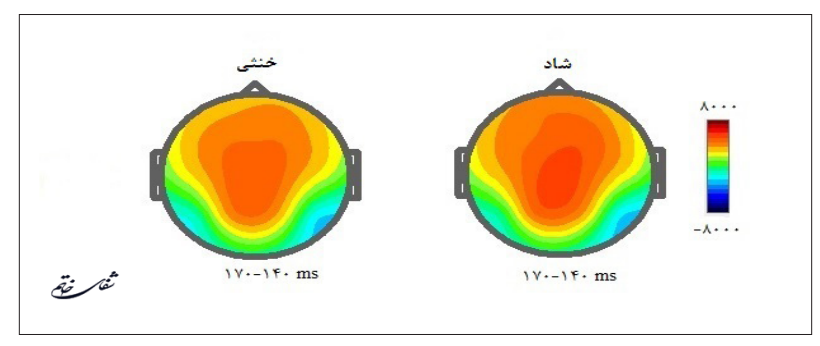

NIV. تصوير 9- توزيع بتانسيل روى سر براى مولفهُ

\footnotetext{
${ }^{18}$ Lateralization

${ }^{19}$ Left hemisphere

${ }^{20}$ Right hemisphere
} 

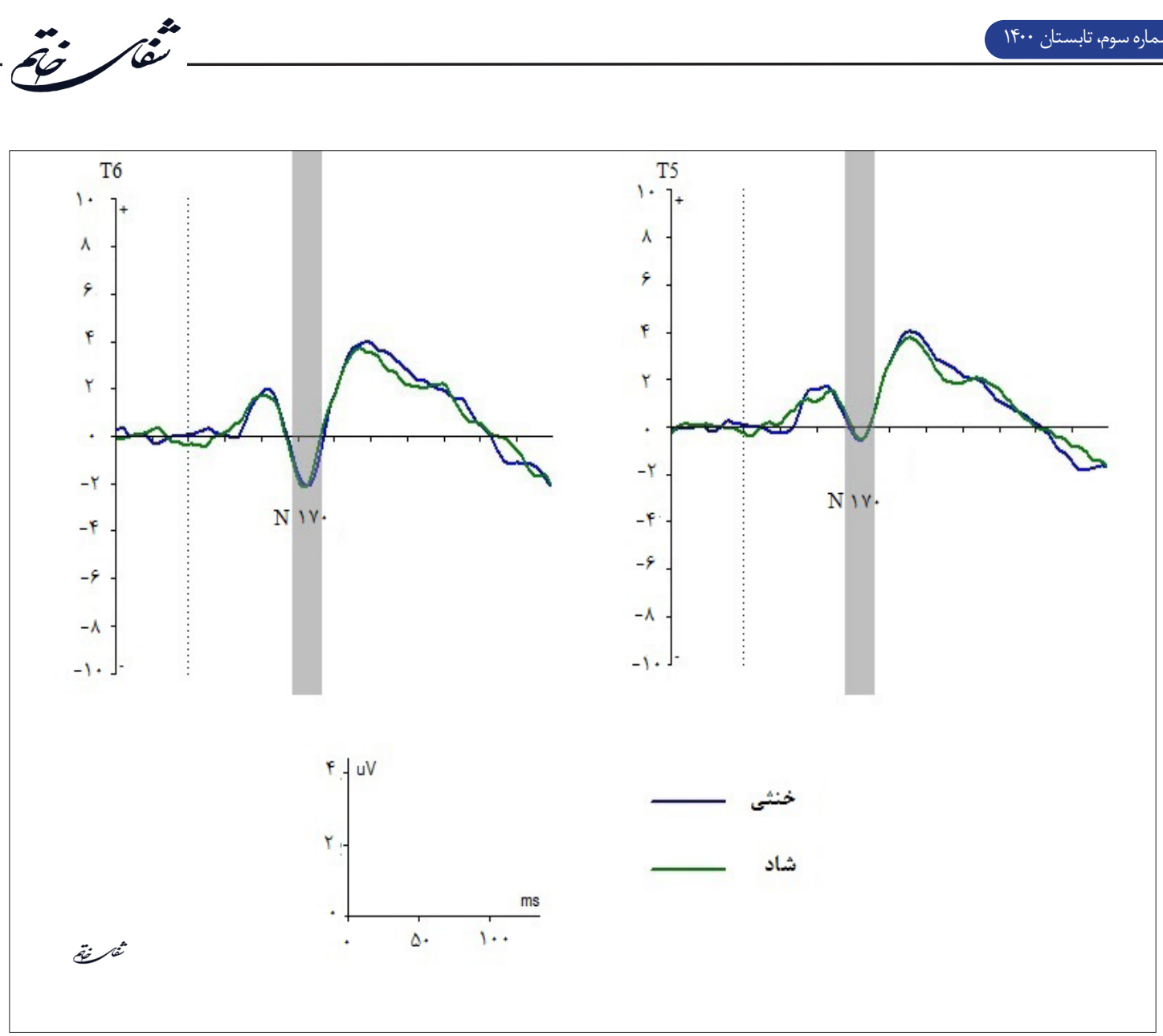

تصوير •|- امواج متوسط بزرك الكترودهاى دو طرف نواحى كيجًاهى (T5,T6) كه نشاندهنده مدولاسيون جانبى •NIV)

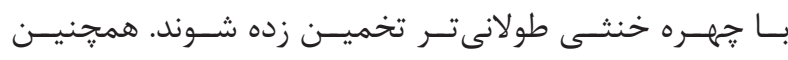

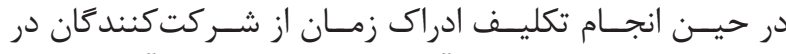

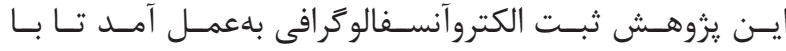

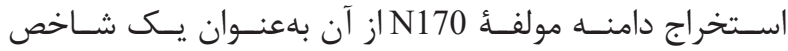

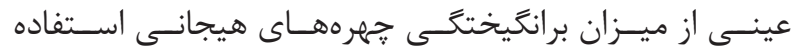

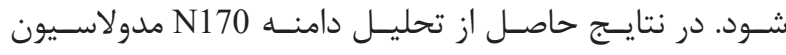

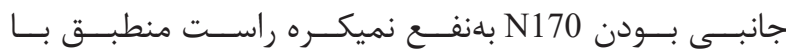

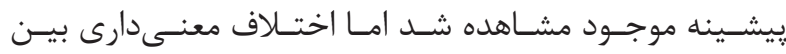

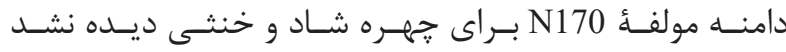

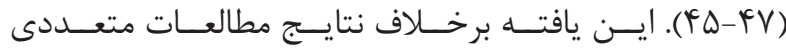

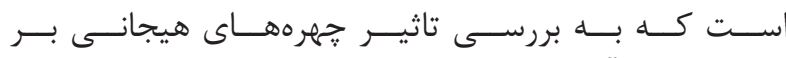

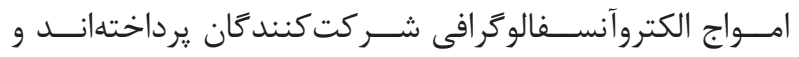

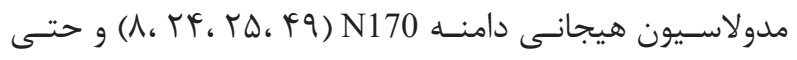

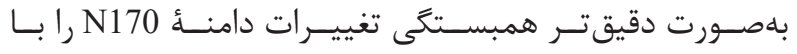

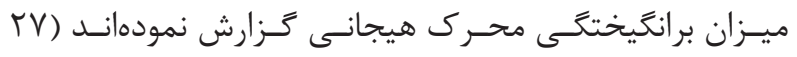

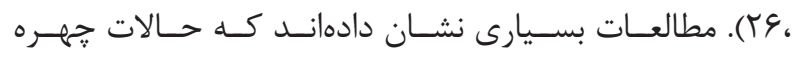

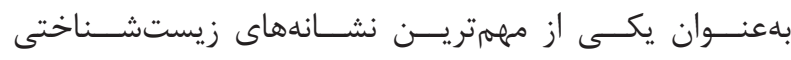

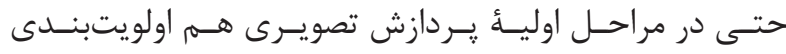

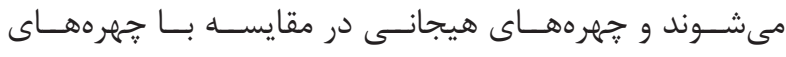

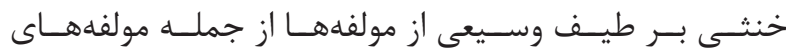

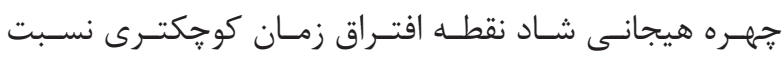

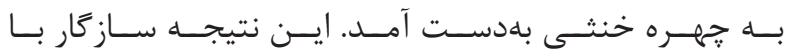

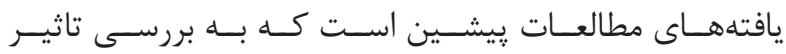

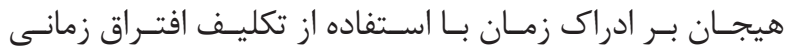

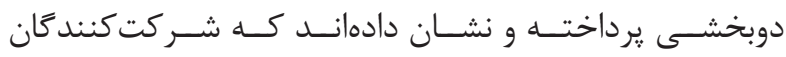

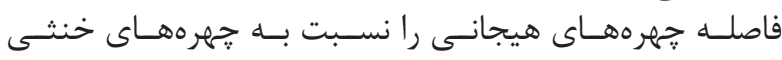

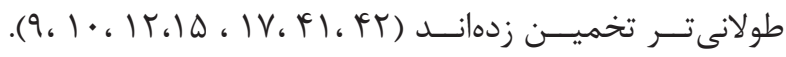

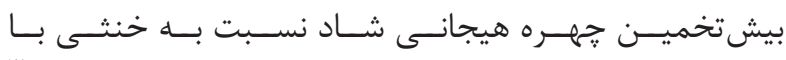

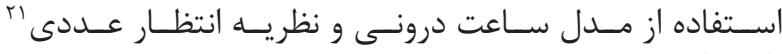
قابـل توضيـح اسـت (SET)

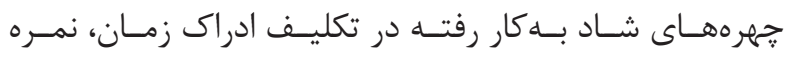

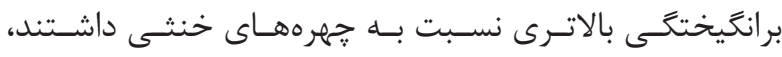

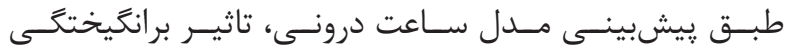

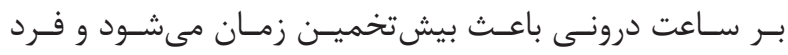

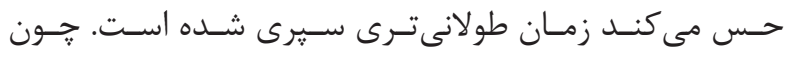

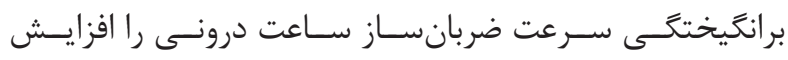

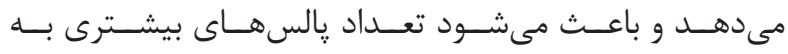

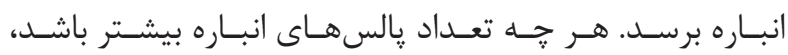

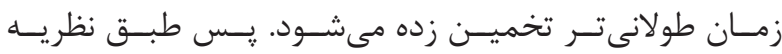

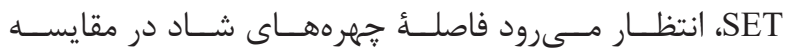




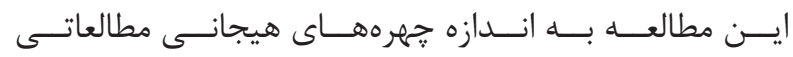

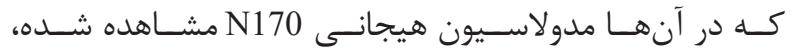

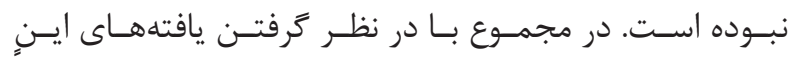

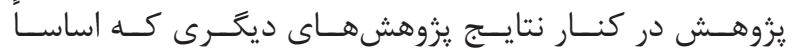

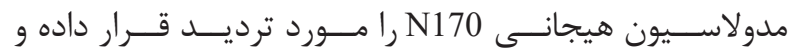

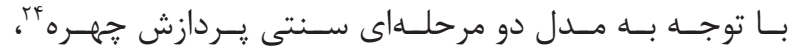

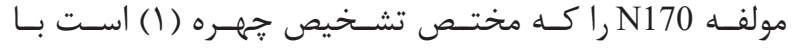

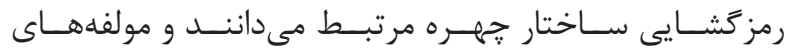

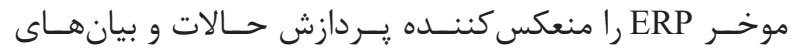

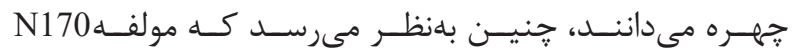

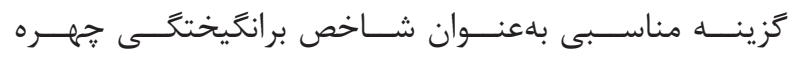

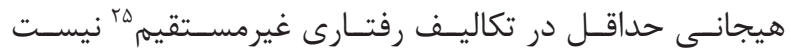

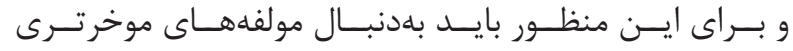

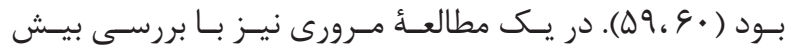

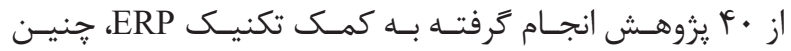

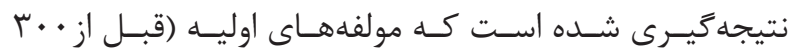

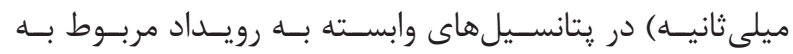

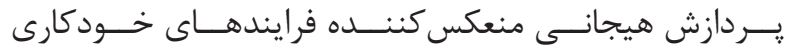

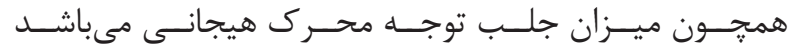

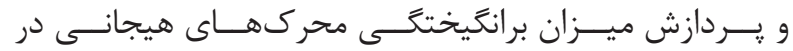

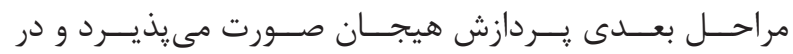

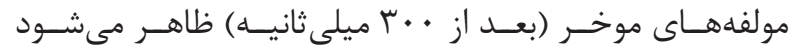

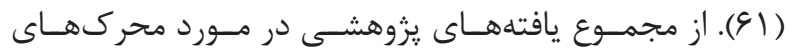

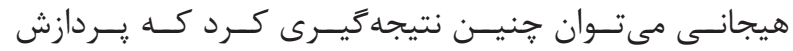

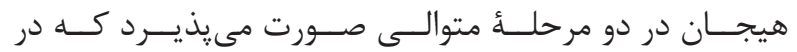

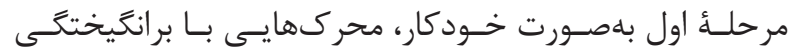

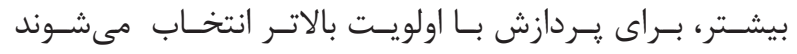

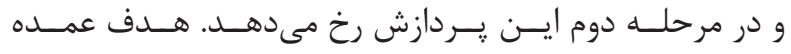

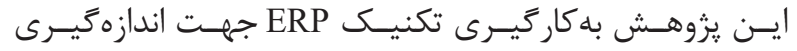

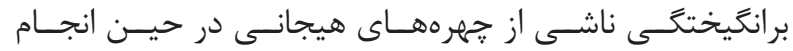

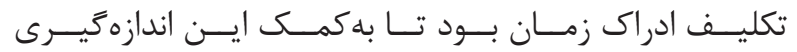

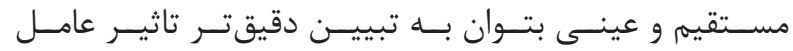

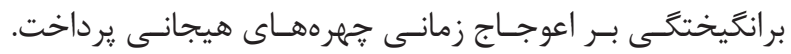

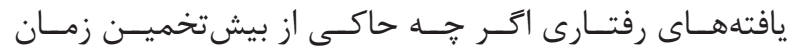

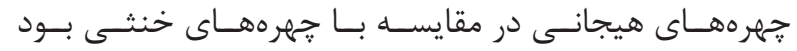

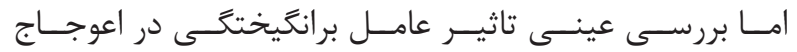

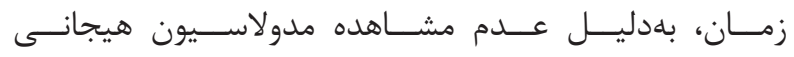

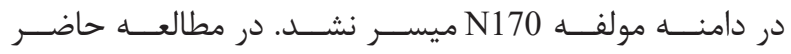

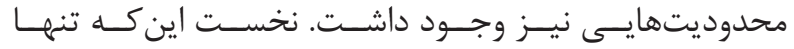

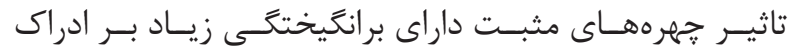

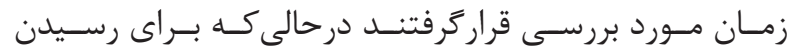

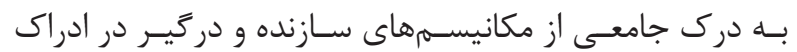

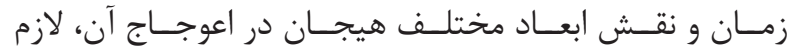

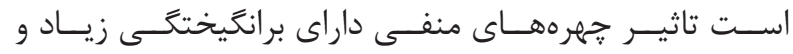

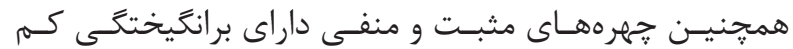

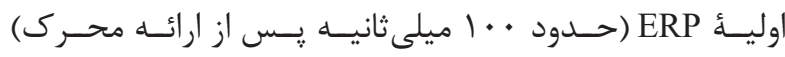

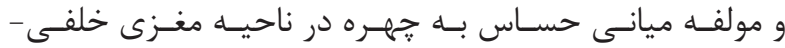

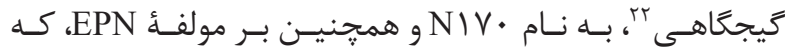

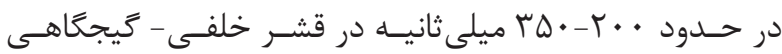

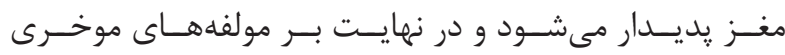

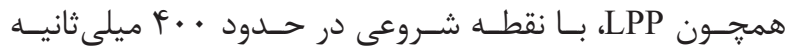

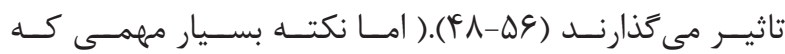

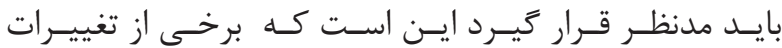

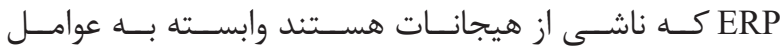

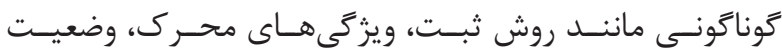

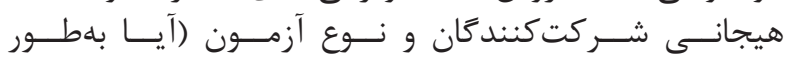

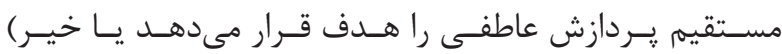

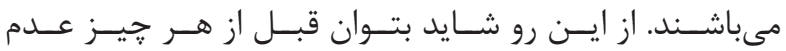

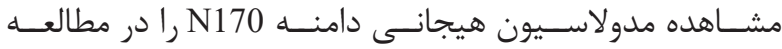

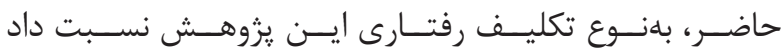

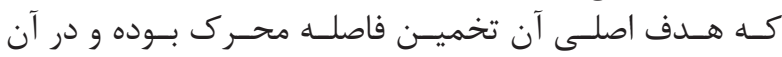

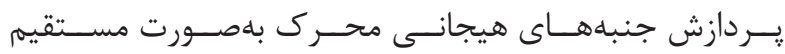

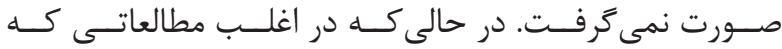

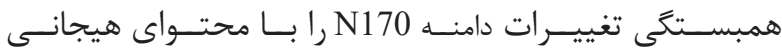

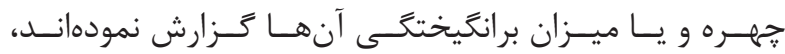

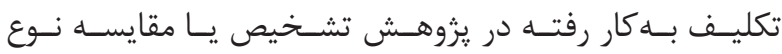

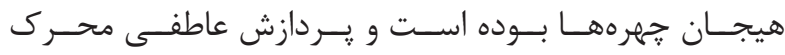

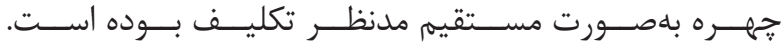

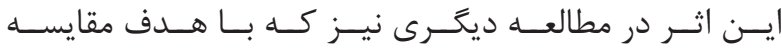

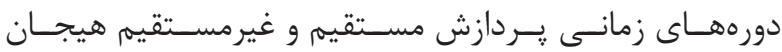

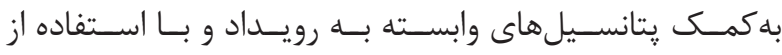

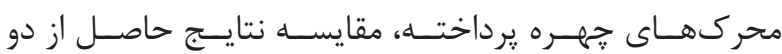

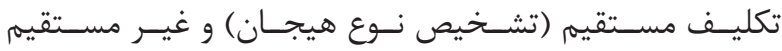

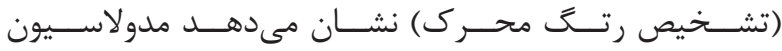

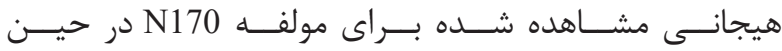

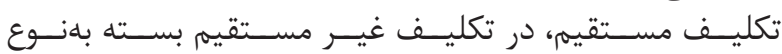

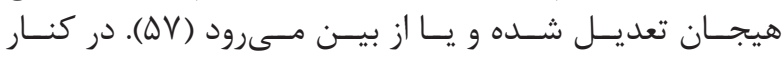

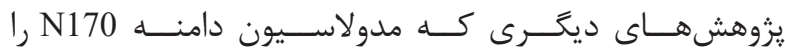

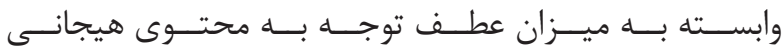

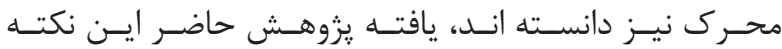

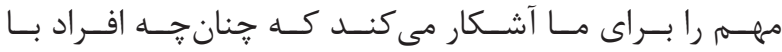

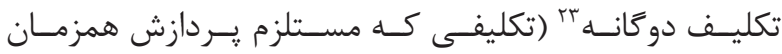

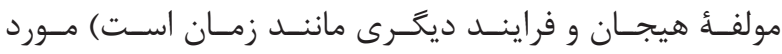

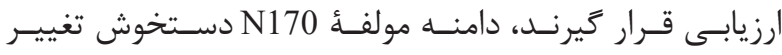

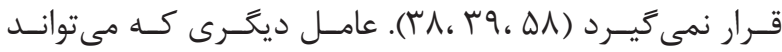

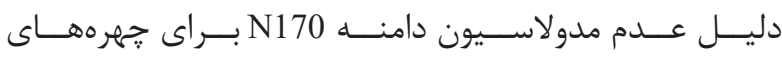

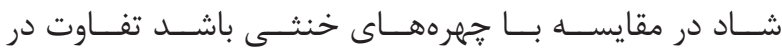

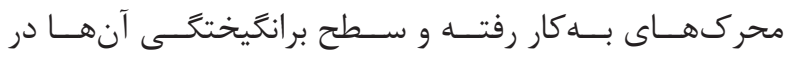

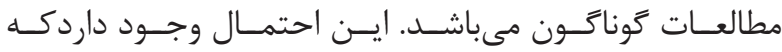

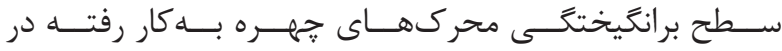




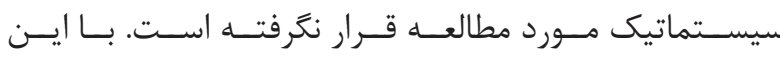

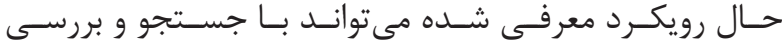

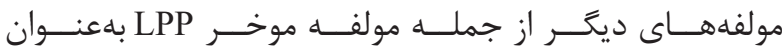

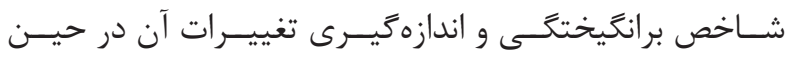

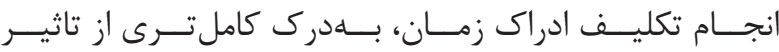

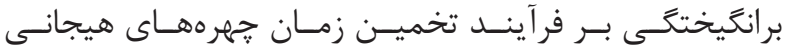

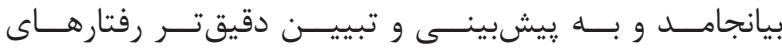

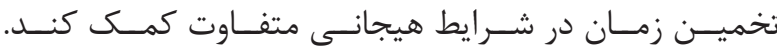

$$
\text { تشكر و قدردانى }
$$

بدينوســيله از مســاعدت اســاتيد محتـــرم دانشـــاه

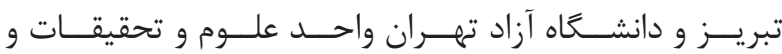

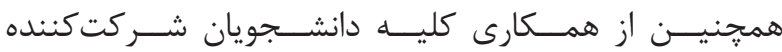

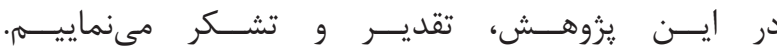

1. Vanderploeg RD, Brown WS, Marsh JT. Judgements of emotion in words and faces: ERP correlates. International Journal of Psychophysiology. 1987; 5(3): 193-205.

2. Gibbon J, Church RM, Meck WH. Scalar timing in memory. Annals of the New York Academy of sciences. 1984; 423(1): 52-77.

3. Wearden JH, Penton-Voak IS. Feeling the heat: Body temperature and the rate of subjective time, revisited. The Quarterly Journal of Experimental Psychology Section B. 1995; 48(2b): 129-41.

4. Maricq AV, Roberts S, Church RM. Methamphetamine and time estimation. Journal of Experimental Psychology: Animal Behavior Processes. 1981; 7(1): 18.

5. Cacioppo JT, Gardner WL. Emotion. Annual review of psychology. 1999; 50(1): 191-214.

6. Tamm M, Uusberg A, Allik J, Kreegipuu K. Emotional modulation of attention affects time perception: Evidence from event-related potentials. Acta psychologica. 2014; 149: 148-56.

7. Angrilli A, Cherubini P, Pavese A, Manfredini S. The influence of affective factors on time perception. Perception \& psychophysics. 1997; 59(6): 972-82.

8. Noulhiane M, Mella N, Samson S, Ragot R, Pouthas V. How emotional auditory stimuli modulate time perception. Emotion. 2007; 7(4): 697.

9. Ishikawa K, Okubo M. Overestimation of the

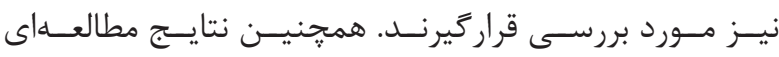

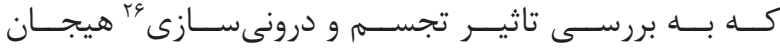

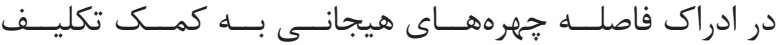

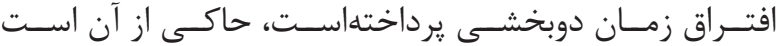

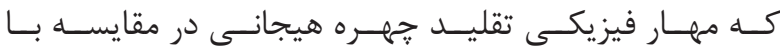

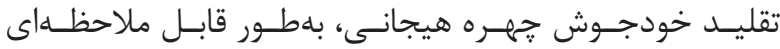

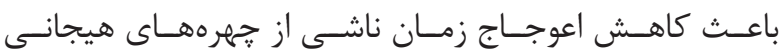

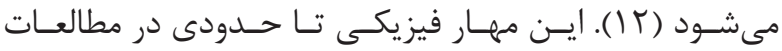

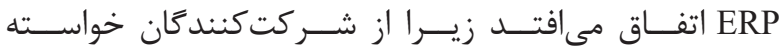

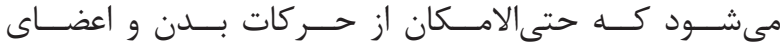

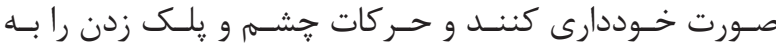

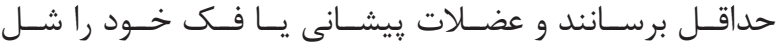

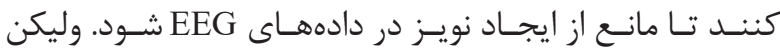

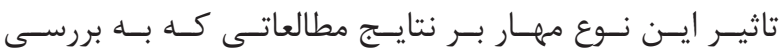

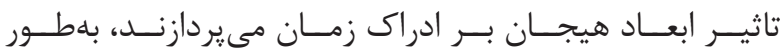

منابع

subjective experience of time in social anxiety: effects of facial expression, gaze direction, and time course. Frontiers in psychology. 2016; 7: 611.

10. Tipples J. Negative emotionality influences the effects of emotion on time perception. Emotion. 2008;8(1):127.

11. Tipples J, Brattan V, Johnston P. Facial emotion modulates the neural mechanisms responsible for short interval time perception. Brain topography. 2015; 28(1): 104-12.

12. Effron DA, Niedenthal PM, Gil S, DroitVolet S. Embodied temporal perception of emotion. Emotion. 2006; 6(1): 1.

13. Jones CR, Gaigg SB, Lambrechts A. Using time perception to explore sensitivity to emotional stimuli in autism spectrum disorder. ProcediaSocial and Behavioral Sciences. 2014; 126: 102-3.

14. Bar-Haim Y, Lamy D, Pergamin L, Bakermans-Kranenburg MJ, Van Ijzendoorn MH. Threat-related attentional bias in anxious and nonanxious individuals: a meta-analytic study. Psychological bulletin. 2007; 133(1): 1.

15. Doi H, Shinohara K. The perceived duration of emotional face is influenced by the gaze direction. Neuroscience letters. 2009; 457(2): 97-100.

16. Droit-Volet S, Brunot S, Niedenthal P. BRIEF REPORT Perception of the duration of emotional events. Cognition and Emotion. 2004 ;18(6): 849-58. 
17. Gil S, Niedenthal PM, Droit-Volet S. Anger and time perception in children. Emotion. 2007; 7(1): 219.

18. Luck S. An introduction to event related potentials and their neural origins. An introduction to the event related potential technique. $2005 ; 11$

19. Rossion B, Joyce CA, Cottrell GW, Tarr MJ. Early lateralization and orientation tuning for face, word, and object processing in the visual cortex. Neuroimage. 2003; 20(3): 1609-24.

20. Allison T, Puce A, Spencer DD, McCarthy G. Electrophysiological studies of human face perception. I: Potentials generated in occipitotemporal cortex by face andnon-facestimuli. Cerebral cortex. 1999;9(5):415-30

21. Ghuman AS, Brunet NM, Li Y, Konecky RO, Pyles JA, Walls SA, et al. Dynamic encoding of face information in the human fusiform gyrus. Nature communications. 2014; 5(1): 1-10.

22. Eimer M. The face-sensitive N170 component of the event-related brain potential. The Oxford handbook of face perception. 2011; 28: 329-44.

23. Rossion B, Jacques C. Does physical interstimulus variance account for early electrophysiological face sensitive responses in the human brain? Ten lessons on the N170. Neuroimage. 2008; 39(4): 1959-79.

24. Bublatzky F, Gerdes A, White AJ, Riemer M, Alpers GW. Social and emotional relevance in face processing: happy faces of future interaction partners enhance the late positive potential. Frontiers in human neuroscience. 2014; 8: 493.

25. Bayer M, Schacht A. Event-related brain responses to emotional words, pictures, and faces-a cross-domain comparison. Frontiers in psychology. 2014; 5: 1106.

26. Almeida PR, Ferreira-Santos F, Chaves PL, Paiva TO, Barbosa F, Marques-Teixeira J. Perceived arousal of facial expressions of emotion modulates the N170, regardless of emotional category: time domain and time-frequency dynamics. International Journal of Psychophysiology. 2016; 99: 48-56.

27. Hietanen JK, Nummenmaa L. The naked truth: the face and body sensitive N170 response is enhanced for nude bodies. PLoS One. 2011; 6(11).
28. Droit-Volet S, Meck WH. How emotions colour our perception of time. Trends in cognitive sciences. 2007; 11(12): 504-13.

29. Gil S, Droit-Volet S. "Time flies in the presence of angry faces" depending on the temporal task used! Acta psychologica. 2011; 136(3): 354-62.

30. Gil S, Droit-Volet S. How do emotional facial expressions influence our perception of time? Attention, representation, and human performance: Integration of cognition, emotion and motivation. 2011:61-74.

31. Gil S, Droit-Volet S. Time perception in response to ashamed faces in children and adults. Scandinavian Journal of Psychology. 2011; 52(2): 138-45.

32. Lundqvist D, Flykt A, Öhman A. The Karolinska directed emotional faces (KDEF). CD ROM from Department of Clinical Neuroscience, Psychology section, Karolinska Institutet. 1998; 91: 630.

33. Bradley MM, Lang PJ. Measuring emotion: the self-assessment manikin and the semantic differential. Journal of behavior therapy and experimental psychiatry. 1994; 25(1): 49-59.

34. Nabizadeh Chianeh G, Vahedi S, Rostami M, Nazari MA. Validity and Reliability of SelfAssessment Manikin. Journal of Research in Psychological Health. 2012;6(2):52-61.

35. Wichmann FA, Hill NJ. The psychometric function: I. Fitting, sampling, and goodness of fit. Perception \& psychophysics. 2001; 63(8): 1293-313.

36. Droit-Volet S, Fayolle S, Gil S. Emotion and time perception in children and adults: the effect of task difficulty. Timing \& Time Perception. 2016; 4(1): 7-29.

37. Fayolle S, Gil S, Droit-Volet S. Fear and time: Fear speeds up the internal clock. Behavioural processes. 2015; 120: 135-40.

38. Eimer M, Holmes A. An ERP study on the time course of emotional face processing. Neuroreport. 2002; 13(4): 427-31.

39. Eimer M, Holmes A. Event-related brain potential correlates of emotional face processing. Neuropsychologia. $\quad 2007 ; \quad 45(1)$ : $15-31$. 


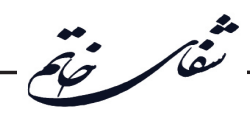

40. Herrmann MJ, Aranda D, Ellgring H, Mueller TJ, Strik WK, Heidrich A, et al. Face-specific event-related potential in humans is independent from facial expression. International Journal of Psychophysiology. 2002; 45(3): 241-4.

41. Gil S, Rousset S, Droit-Volet S. How liked and disliked foods affect time perception. Emotion. 2009; 9(4): 457.

42. Kliegl KM, Limbrecht-Ecklundt K, Dürr L, Traue HC, Huckauf A. The complex duration perception of emotional faces: effects of face direction. Frontiers in psychology. 2015; 6: 262.

43. Gibbon J. Scalar expectancy theory and Weber's law in animal timing. Psychological review. 1977; 84(3): 279.

44. Johnston VS, Miller DR, Burleson MH. Multiple $\mathrm{P} 3 \mathrm{~s}$ to emotional stimuli and their theoretical significance. Psychophysiology. 1986; 23(6): 684-94.

45. Mercure E, Dick F, Johnson MH. Featural and configural face processing differentially modulate ERP components. Brain research. 2008; 1239: 162-70.

46. Scott LS, Nelson CA. Featural and configural face processing in adults and infants: A behavioral and electrophysiological investigation. Perception. 2006; 35(8): 1107-28.

47. Wang H, Sun P, Ip C, Zhao X, Fu S. Configural and featural face processing are differently modulated by attentional resources at early stages: an eventrelated potential study with rapid serial visual presentation. Brain research. 2015; 1602: 75-84.

48. Ashley V, Vuilleumier P, Swick D. Time course and specificity of event-related potentials to emotional expressions. Neuroreport. 2004 ;15(1): 211-6.

49. Blau VC, Maurer U, Tottenham N, McCandliss BD. The face-specific N170 component is modulated by emotional facial expression. Behavioral and brain functions. 2007; 3(1): 7 .

50. Adolphs R. Recognizing emotion from facial expressions: psychological and neurological mechanisms. Behavioral and cognitive neuroscience reviews. 2002; 1(1): 21-62.
51. Batty M, Taylor MJ. Early processing of the six basic facial emotional expressions. Cognitive Brain Research. 2003; 17(3): 613-20.

52. Eger E, Jedynak A, Iwaki T, Skrandies W. Rapid extraction of emotional expression: evidence from evoked potential fields during brief presentation of face stimuli. Neuropsychologia. 2003; 41(7): 808-17.

53. Pizzagalli D, Regard M, Lehmann D. Rapid emotional face processing in the human right and left brain hemispheres: an ERP study. Neuroreport. 1999; 10(13): 2691-8.

54. Williams LM, Palmer D, Liddell BJ, Song L, Gordon E. The 'when' and 'where' of perceiving signals of threat versus non-threat. Neuroimage. 2006; 31(1): 458-67.

55. Schacht A, Sommer W. Emotions in word and face processing: early and late cortical responses. Brain and cognition. 2009; 69(3): 538-50.

56. SchuppH, CuthbertB, Bradley M, HillmanC,Hamm A, LangP.Brainprocessesinemotionalperception:Motivated attention. Cognition and emotion. 2004; 18(5): 593-611.

57. Frühholz S, Jellinghaus A, Herrmann M. Time course of implicit processing and explicit processing of emotional faces and emotional words. Biological psychology. 2011; 87(2): 265-74.

58. Yoncheva YN, Blau VC, Maurer U, McCandliss BD. Attentional focus during learning impacts N170 ERP responses to an artificial script. Developmental neuropsychology. 2010; 35(4): 423-45.

59. Pascalis O, de Martin de Viviés X, Anzures G, Quinn PC, Slater AM, Tanaka JW, et al. Development of face processing. Wiley Interdisciplinary Reviews: Cognitive Science. 2011; 2(6): 666-75.

60.Tsao DY, Livingstone MS. Mechanisms of face perception. Annu Rev Neurosci. 2008; 31: 411-37.

61.Olofsson JK, Nordin S, Sequeira H, Polich J. Affective picture processing: an integrative review of ERP findings. Biological psychology. 2008; 77(3): 247-65. 